\title{
Estimating neuronal conductance model parameters using dynamic action potential clamp
}

Deerasooriya Y.a, Berecki G. ${ }^{b}$, Kaplan D. ${ }^{b}$, Forster I.C. ${ }^{b}$, Halgamuge S. ${ }^{a, c}$, and Petrou S. ${ }^{b, d, e,{ }^{*}}$

aDepartment of Mechanical Engineering, The University of Melbourne, Parkville, Victoria, Australia.

${ }^{b}$ Ion Channels and Disease Group, The Florey Institute of Neuroscience and Mental Health, The University of Melbourne, Parkville, Victoria, Australia.

${ }^{\mathrm{C}}$ Research School of Engineering, College of Engineering \& Computer Science, The Australian National University, Canberra, Australian Capital Territory, Australia.

dDepartment of Medicine, Royal Melbourne Hospital, The University of Melbourne, Parkville, Victoria, Australia.

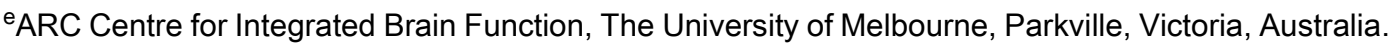

*Correspondence: spetrou@unimelb.edu.au 


\section{ABSTRACT}

Background Parameterization of neuronal membrane conductance models relies on data acquired from current clamp (CC) or voltage clamp (VC) recordings. Although the CC approach provides key information on a neuron's firing properties, it is often difficult to disentangle the influence of multiple conductances that contribute to the excitation properties of a real neuron. Isolation of a single conductance using pharmacological agents or heterologous expression simplifies analysis but requires extensive $\mathrm{VC}$ evaluation to explore the complete state behavior of the channel of interest.

New Method We present an improved parameterization approach that uses data derived from dynamic action potential clamp (DAPC) recordings to extract conductance equation parameters. We demonstrate the utility of the approach by applying it to the standard Hodgkin-Huxley conductance model although other conductance models could be easily incorporated as well.

Results Using a fully simulated setup we show that, with as few as five action potentials previously recorded in DAPC mode, sodium conductance equation parameters can be determined with average parameter errors of less than $4 \%$ while action potential firing accuracy approaches $100 \%$. In real DAPC experiments, we show that by "training" our model with five or fewer action potentials, subsequent firing lasting for several seconds could be predicted with $\sim 96 \%$ mean firing rate accuracy and $94 \%$ temporal overlap accuracy.

Comparison with Existing methods Our DAPC-based approach surpasses the accuracy of VCbased approaches for extracting conductance equation parameters with a significantly reduced temporal overhead.

Conclusion DAPC-based approach will facilitate the rapid and systematic characterization of neuronal channelopathies. 


\section{KEYWORDS}

Ion channel biophysics; Conductance modelling; Hodgkin and Huxley; Dynamic action potential clamp 


\section{Highlights}

- An improved approach to parameterize neuronal conductance models is described.

- The approach allows precise estimation of Hodgkin-Huxley model parameters.

- Firing patterns of real action potentials were predicted with high accuracy.

- Experimental time is significantly reduced compared with voltage clamp methods. 


\section{INTRODUCTION}

Mathematical models are frequently used to study the dynamics of neurons and their networks under a broader range of conditions compared to those feasible with experimental approaches (1-5). For example, the classical Hodgkin Huxley ( $\mathrm{HH}$ ) equations (6) are widely used for developing biophysically realistic ion channel conductances that underlie firing in neuronal models $(2,7,8)$. Specifically, HH equations were developed to describe the membrane conductance of the squid giant axon and they have been successfully used to parameterize mammalian neuronal conductances (9-13) or cells that heterologously express a desired channel type $(1,14,15)$. Current clamp (CC) of real neurons provides a direct measure of action potential (AP) properties that are useful for constraining the $\mathrm{HH}$ equations and refining model behavior. However, this approach alone is limited because of the need to separate the contributions to excitability and AP firing behavior made by the multiple ion channel conductances found in neurons $(12,16,17)$. Isolation of the conductance of interest using either a pharmacological approach in neurons or controlled heterologous expression, enables direct investigation of the conductance, but typically such experiments are limited to VC recordings. Although the traditional VC approach provides accurate control of the membrane potential, it requires exploration of channel's state transitions using extensive experimental protocols to acquire the data needed to parameterize the model equations. VC protocols typically involve a sequence of voltage steps by means of which the initial states of the channels are predefined and the transitions to other states investigated following a test voltage step. As such these protocols do not represent the 'natural' time course of membrane potential changes of excitable cells during the AP.

The dynamic clamp (DC) approach was implemented in recent studies to acquire data from neurons for modeling ion channel conductances $(11,18)$. This involves first, neuronal recording, i.e., recording APs from a neuron containing the channel conductance of interest. The channel is then pharmacologically blocked and functionally replaced (in real time) using a DC-injected conductance model developed in silico. The parameters for this model are iteratively fine-tuned in real time until APs appear and match the originally recorded firing data (11). Because of the non-stationarity of neuronal recordings, a priori knowledge from another recording session is needed to estimate initial parameter values and constraints are imposed to enable convergence of the model within periods where the recorded neuron is stable. To overcome these limitations, we have developed a parameterization strategy based on the dynamic action potential clamp (DAPC) (19) for studying an isolated conductance under voltage clamp conditions in the context of biologically realistic membrane potential dynamics. A key advantage of the DAPC method is that fitting the AP signal can be done post hoc, without the need for consideration of non-stationarity of neuronal recordings, hence a priori parameter values are not required.

The DAPC technique is an extension of the DC approach (20) and involves establishing a 
real-time feedback loop between a biological cell and an in silico neuron to create a 'hybrid' neuron. An important difference between the DC and DAPC approaches is that rather than pharmacologically removing and then replacing the conductance of interest by in silico algorithms as in the former case, here we heterologously express the ion channel conductance in a mammalian cell and combine it with additional in silico modeled conductances to restore AP firing. Unlike standard VC protocols, the DAPC approach generates physiologically relevant data and is able to predict the effect of changes in ion channel properties on neuronal excitability and subsequent AP firing in real-time. To this end, we have employed improved model fitting approaches that are not limited to the time periods of stationary behavior of the recorded neuron. Moreover, the method described here is applicable to conductances isolated from neurons as well as those expressed in cell lines. To validate this strategy, we used computer simulations and in vitro experimental data obtained from Chinese hamster ovary (CHO) cells transiently expressing neuronal $\mathrm{Na}_{\mathrm{v}} 1.2$ channels. We chose the standard $\mathrm{HH}$ formulation to define the voltage dependence of conductances, although our general strategy would be applicable to other channel gating models. Indeed, the $\mathrm{HH}$ formulation used here was considered areasonable compromise between accuracy and biological realism as compared to biophysically strict Markov or purely phenomenological formalisms (e.g., ref. (21)). To verify the robustness of the DAPC approach, we also compared the efficiency in estimating $\mathrm{HH}$ parameter values when using different stimulating signals to perturb the conductances under DAPC conditions. Additionally, we propose an improved optimization method to increase the accuracy of the model parameters in the presence of noise. 


\section{MATERIALS AND METHODS}

\subsection{Channel models based on the HH formulation}

The total membrane current $(I)$ is given by:

$$
\begin{aligned}
& I=C_{m} \frac{d V(t)}{d t}+I_{\text {leak }}+\sum I_{\text {ion }} \\
& I_{\text {leak }}=G_{\text {leak }}\left(V-V_{\text {leak }}\right) \\
& I_{\text {ion }}=G_{\text {ion }}\left(V-V_{\text {ion }}\right)
\end{aligned}
$$

$C_{m}$ is the membrane capacitance; $I_{\text {leak }}$ is the leak current; $I_{\text {ion }}$ is the ion channel current; $G_{\text {leak }}$ is the leak conductance; $V_{\text {leak }}$ is the leak reversal potential; $G_{\text {ion }}$ is the maximum ion channel current; $P_{o}$ is the open channel probability and $V_{\text {ion }}$ is the reversal potential of the ion.

For modeling the leak current equation 2 was used with the following parameter values: $G_{\text {leak }}=$ $0.3 \mathrm{mScm}^{-2}$ and $v_{\text {leak }}=-54.3 \mathrm{mV}$.

For modeling the sodium channel open probability in fully simulated experiments, the standard HH formulation was used (6), designated as the Type 1 sodium channel model. The channel open probability $\left(p_{o}\right)$ is given by:

$$
\begin{aligned}
& p_{o}=m^{3} h \\
& \frac{d m}{d t}=\frac{m_{\infty}(V)-m}{\tau_{m}(V)} \\
& \frac{d h}{d t}=\frac{h_{\infty}(V)-h}{\tau_{h}(V)}
\end{aligned}
$$

where $m$ and $h$ represent the activation and inactivation of sodium gating particles, respectively; $m_{\infty}$ and $h_{\infty}$ are the corresponding steady-state values and $\tau_{m}$ and $\tau_{h}$ are the corresponding time constants, expressed as (1),

$$
m_{\infty}=\frac{1}{1+\exp \left(s_{m}\left(V-V_{0.5, m}\right)\right)}
$$




$$
\begin{aligned}
h_{\infty} & =\frac{1}{1+\exp \left(s_{h}\left(V-V_{0.5, h}\right)\right)} \\
\tau_{m} & =a_{m} \exp \left(-b_{m} V\right) \\
\tau_{h} & =a_{h} \exp \left(-b_{h} V\right)
\end{aligned}
$$

The parameter values were taken from (6) and modified to represented in the general formulation given above $\left(G_{N a}=120 \mathrm{mScm}^{-2}\right.$ (maximum conductance), $S_{m}=-0.115031 \mathrm{mV}^{-1}$ (slope of steady state activation), $V_{0.5, m}=-40.025 \mathrm{mV}$ (activation half-voltage), $a_{m}=0.20579 \mathrm{~ms}, b_{m}=0.005575$ $\mathrm{mV}^{-1}$ (coefficients of activation time constant), $s_{h}=0.083166 \mathrm{mV}^{-1}$ (slope of steady-state inactivation), $V_{0.5, h}=-62.308 \mathrm{mV}$ (Inactivation half voltage), $a_{h}=1.139939 \mathrm{~ms}, b_{h}=0.015814$ $\mathrm{mV}^{-1}$ (coefficients of activation time constant), $V_{N a}=50 \mathrm{mV}$ (sodium reversal potential).

For modeling the potassium channel open probability in fully simulated experiments, the following $\mathrm{HH}$ equations were used and this was designated as a Type 1 potassium channel model.

$$
\begin{aligned}
& p_{o}=n^{4} \\
& \frac{d n}{d t}=\frac{n_{\infty}(V)-n}{\tau_{n}(V)}
\end{aligned}
$$

where $n$ represents the activation of potassium gating particle; $n_{\infty}$ and $\tau_{n}$ are steady-state value and time constant of $n$, respectively and are expressed as (1).

$$
\begin{aligned}
& n_{\infty}=\frac{1}{1+\exp \left(s_{n}\left(V-V_{0.5, n}\right)\right)} \\
& \tau_{n}=a_{n} \exp \left(-b_{n} V\right)
\end{aligned}
$$

The parameter values in Eqs. 13 and 14 are based on literature data describing the conductances in the squid giant axon (6). $\left(G_{K}=36 \mathrm{mScm}^{-2}, s_{n}=-0.17064 \mathrm{mV}^{-1}, \mathrm{v} 0.5_{n}=-56.4823\right.$ $\left.\mathrm{mV}, a_{n}=2.58049 \mathrm{~ms}, b_{n}=0.017282 \mathrm{mV}^{-1}, v_{K}=-77 \mathrm{mV}\right)$.

For experiments performed with Nav variants expressed in $\mathrm{CHO}$ cells, a second model was implemented with voltage dependent kinetics to reflect the persistent current components that are not fully inactivated at steady state $(22,23)$ and to avoid time constants from approaching zero at higher 
voltages. For parameterization, the real sodium channel conductance was replaced with a model based on the Type 1 sodium channel formalism above, and this was designated the Type 2 model. The equations for $m_{\infty}, h_{\infty}, \tau_{m}$ and $\tau_{h}$ are given by:

$$
\begin{aligned}
& m_{\infty}=\frac{1}{1+\exp \left(s_{m}\left(V-V_{0.5, m}\right)\right)} \\
& h_{\infty}=\text { base }_{h}+\frac{\left(1-\text { base }_{h}\right)}{1+\exp \left(s_{h}\left(V-V_{0.5, h}\right)\right)} \\
& \tau_{m}=\tau_{m}^{0}+a_{m} \exp \left(-b_{m} V\right) \\
& \tau_{h}=\tau_{h}^{0}+a_{h} \exp \left(-b_{h} V\right)
\end{aligned}
$$

Where base $_{h}$ is the minimum inactivation $\tau^{0} m$ and $\tau^{0} h$ are the time constants for activation and inactivation, respectively for large $V$. For the potassium conductance in the $\mathrm{Na}_{\mathrm{v}}$ experiments above, we used the model implemented by Hu et al. (2) as below and defined as a Type 2 potassium channel model.

$$
\begin{aligned}
& I_{K}=G_{K} n\left(V-V_{K}\right) \\
& \frac{d n}{d t}=\alpha_{n}(1-n)+\beta_{n} n \\
& I_{\text {leak }}=G_{\text {leak }}\left(V-V_{\text {leak }}\right) \\
& \alpha_{n}=\frac{0.02(V-25)}{1-\exp \left(\frac{V-25}{9}\right)} \\
& \beta_{n}=\frac{-0.002(V-25)}{1-\exp \left(\frac{V-25}{9}\right)}
\end{aligned}
$$

\subsection{Modeling approaches based on VC data}

The cell was voltage clamped and the activation and inactivation protocols were applied to acquire VC data for modeling. The activation protocol comprised $100 \mathrm{~ms}$ voltage steps from -70 to $+20 \mathrm{mV}$ in $5 \mathrm{mV}$ increments from a holding potential of $-80 \mathrm{mV}$ (e.g. Fig 2a). The inactivation protocol included 100-ms test voltage steps from $-120 \mathrm{mV}$ to $+20 \mathrm{mV}$ in $5 \mathrm{mV}$ increments followed by a step to $-15 \mathrm{mV}$ to test channel availability (e.g. Fig $2 b)$ (1). 
Two existing approaches based on VC were developed for comparison with the proposed DAPC method. The standard fitting method based on VC data was modified from the method used by Dougalis et al. (24). When calculating the parameters of the standard model, the following assumptions were made:

1. Activation is zero at the holding potential in the activation protocol, i.e. $m(0)=0$.

2. For membrane potentials more positive than $-35 \mathrm{mV}$, channels are inactivated at steady state, i.e. $\mathrm{h}_{\infty}=0$ in the activation protocol.

3. Due to the slower kinetics of inactivation compared to activation, channel inactivation does not start until the conductance becomes maximum.

The maximum conductance values for each test potential in the activation and inactivation protocols were calculated, normalized and fitted against membrane potential to estimate the steadystate curves and related parameters (Eqs. 7 and 8). Then, the activation and inactivation time constants for each membrane potential were estimated by fitting activation conductance curves. Finally, the voltage dependence of activation and inactivation kinetics was determined by fitting these time constant values as a function of membrane potential (Eqs. 9 and 10).

The improved VC fitting method employs the approach used by Thomas et al. (1). This method avoids the errors introduced by assumption 3 above and takes account of the comparable time scales of fast activation and inactivation. For this method, the current trace in each voltage step was fitted with the equation,

$$
I_{\text {ion }}=G_{\text {ion }}\left(m_{\infty}(V)\right)^{3}\left(1+\exp \left(\frac{-t}{\tau_{m}(V)}\right)\right)^{3} \exp \left(\frac{-t}{\tau_{h}(V)}\right)\left(V-V_{\text {ion }}\right)
$$

and the steady-state activation voltage dependence, time constants of activation and inactivation were obtained. The steady-state inactivation curve was obtained from the inactivation protocol traces using a method similar to the standard approach. Then the steady-state and time constant parameters were determined by fitting Eqs. 7-10 to these curves, respectively. Finally, the parameters were further optimized by fitting to all activation and inactivation current traces at once, utilizing the above estimated parameter values as starting points.

\subsection{DAPC modeling approach}

DAPC-based modeling involves connecting a biological conductance provided by a cell expressing the ion channels of interest to virtual (in silico) macroscopic conductances, to generate a hybrid neuron capable of firing APs. $\mathrm{Na}_{\mathrm{v}} 1.2$ channels were expressed in Chinese hamster ovary (CHO) 
cells. Macroscopic sodium currents through $\mathrm{Na}_{\mathrm{v}} 1.2$ channels $\left(\mathrm{I}_{\mathrm{Nav1} .2}\right)$ were recorded using whole-cell patch clamp in VC mode (Fig 1a). The VC amplifier was connected to a real-time computing platform (ADwin Pro II, Jäger Computergesteuerte Messtechnik GmbH, Lorsch, Germany ) to form a realtime closed loop DAPC (Fig 1b), in which the $\mathrm{I}_{\text {Nav1.2 }}$ from the $\mathrm{CHO}$ cell served as input to a computational model, whereas the calculated membrane potential $\left(\mathrm{V}_{\mathrm{m}}\right)$ of the hybrid cell is the output and represents the VC command potential applied to the $\mathrm{CHO}$ cell. For each computational time step (see Section 2.7), the model outputs a new $V_{m}$ value. The behavior of the hybrid neuron was systematically explored in silico by using various stimulating strategies (e.g. synaptic noise) (Fig 1b). DAPC data were used for post hoc optimization and parameterization. In this workflow shown in Figure 1c, the $\mathrm{HH}$ conductance model for the expressed channels was parameterized according to a three step process comprising: (1) substitution of the cell's conductance with a standard HH model by using initial parameters generated randomly within predefined limits within two-orders greater or smaller than published data; (2) use of the parameters in the all in silico simulation of the DAPC for predicting AP firing; (3) generate a new parameter set by minimizing the error between the DAPC previously recorded voltage traces and those obtained with the all in silico model. The computational cycles between steps 2 and 3 were repeated multiple times until the stopping criteria defined by the fitness function were reached (see Section 2.4). 
a) Conventional VC

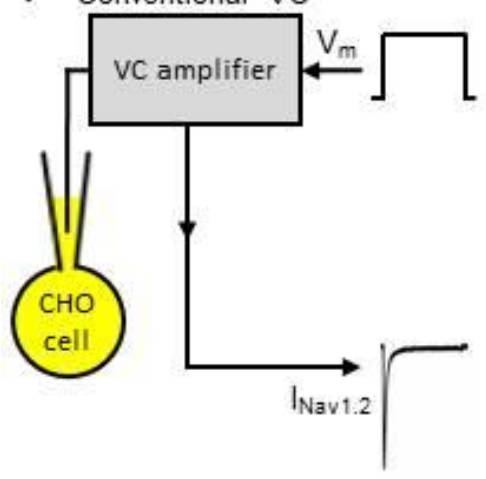

b)

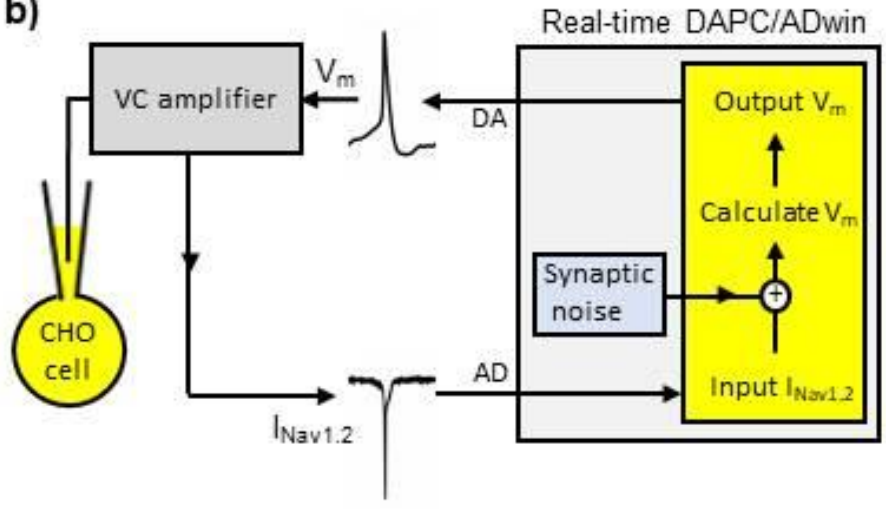

c)

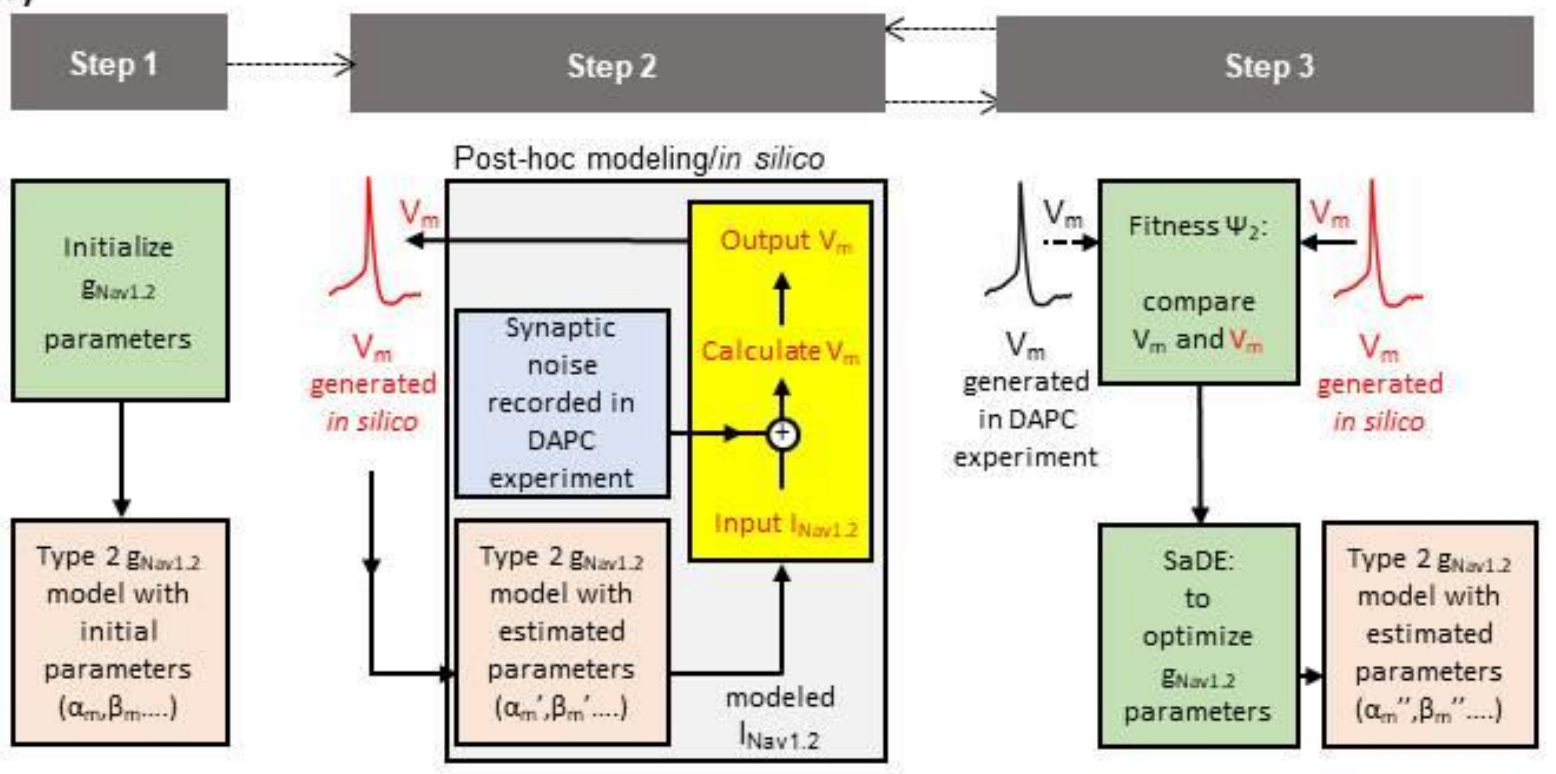

Figure 1. Dynamic action potential clamp (DAPC) modeling approach to parameterize conductance models. (a) Conventional VC setup: The ion channel under investigation is expressed in a CHO cell line and is whole-cell voltage clamped to record the macroscopic sodium current $\left(\mathrm{I}_{\mathrm{Nav1.2}}\right)$. (b) DAPC experimental setup: $\mathrm{CHO}$ cell current $\left(\mathrm{I}_{\mathrm{Nav1.2}}\right)$ is recorded using whole-cell patch clamp in conventional VC mode. The recorded $\mathrm{I}_{\mathrm{Nav1.2}}$ is used as an input to in silico computational model cell (yellow-colored rectangle), which is used to compute $\mathrm{V}_{\mathrm{m}}$ of the 'hybrid' cell. The output $\mathrm{V}_{\mathrm{m}}$ is applied as the $\mathrm{VC}$ command to the CHO cell to establish real-time DAPC recording mode. Stimuli (e.g. current step or synaptic noise) are applied to evoke APs in this hybrid neuron model. (c) Post hoc parameterization and optimization of a conductance model using DAPC data: the macroscopic $\mathrm{I}_{\mathrm{Nav1.2}}$ is substituted with a sodium conductance model with unknown parameters establishing an all in silico model. An optimization algorithm is used to estimate the sodium conductance parameters in the all in silico model that minimizes the error between observed and modeled data. 


\subsection{Fitness function and optimization procedures}

To parameterize the above models, two optimization processes were used. The one step procedure employs the sum of point-wise square error (SSE) for the DAPC and fully in silico model data.This method uses $\psi_{1}$ as the fitness function (see below) and is referred to as the one-step method.

The two-step procedure takes account of the effects of noise, assessed by calculating the signal to noise ratio (SNR) with the following equation:

$$
S N R(d B)=20 \log \left(\frac{\mu_{A}}{\sigma_{\text {Noise }}}\right)
$$

where $\mu_{A}$ is the maximum ion channel current (during an $\mathrm{AP}$ ) averaged over multiple APs in the trace and $\sigma_{\text {Noise }}$ is the noise standard deviation. As for the one-step optimization method, parameters were iteratively estimated using the fitness function $\psi 1$ in the first step, however for this implementation a more relaxed stopping criterion was used. The first step 'explores' the parameter space to identify a particular region with a desirable fitness range. The final population of parameters in the first step was used as the initial population for the second step where optimization continued with fitness function $\psi_{2}$. This step 'exploits' the parameter space already narrowed by the first step to estimate the exact parameter set that gives the best fitness. This process was continued until the fitness value did not improve within a given tolerance over a number of consecutive iterations.

Whole-cell current recorded during DAPC recording mode is filtered by virtue of the capacitance terms in the equations used to integrate current into membrane voltage. Therefore, the membrane potential data will be intrinsically less noisy than membrane current data and is a better signal for optimization. Taking this into consideration, two types of fitness functions $(\psi)$ were proposed:

$$
\begin{gathered}
\varphi_{1}=\sum_{i}\left(V_{\text {exp }}^{i}-V_{\text {mod }}^{i}\right)^{2} \\
\varphi_{2}=\sum_{i}\left(V_{A P, \text { exp }}^{i}-V_{A P, \text { mod }}^{i}\right)^{2}+w_{A P} \sum_{i}\left(t_{\text {APinit }, \exp }^{i}-t_{\text {APinit }, \text { mod }}^{i}\right)^{2}
\end{gathered}
$$

where $V_{\text {exp }}$ and $V_{\text {mod }}$ are the complete membrane potential traces from DAPC experiments and the insilico model. $V_{A P \exp }$ and $V_{A P m o d}$ are the AP morphology data without sub-threshold voltages data from DAPC experiments and in-silico model. $t_{\text {APtimeexp }}$ and $t_{\text {APtimemod }}$ are the AP timing data from DAPC experiments and in-silico model. $W_{A P}$ is the weight of AP morphology in the fitness function. The AP 
morphology was extracted from entire membrane potential traces using simple threshold detection. Here, we used the weight $W_{A P}=1$ to provide equal weighting for both AP timing and morphology.

The Self-adaptive Differential Evolution (SaDE) algorithm introduced by Qin et al. (25), a variant of differential evolution algorithms, was used for optimization. Differential evolution is a stochastic, population-based optimization technique inspired by the natural evolution process. It uses selection, mutation and crossover operations to produce the parameter vectors for the next iteration. In $\mathrm{SaDE}$, the initial parameter vector population is generated randomly within the physiologically feasible parameter range. At each iteration, four different mutation strategies are used to mutate the current parameter vectors to generate probable trial parameter vectors for the next iteration:

$$
u_{i, j}=\left\{\begin{array}{c}
x_{r 1, j}+F \cdot\left(x_{r 2, j}-x_{r 3, j}\right), \quad \text { if rand }[0,1)<C R \text { or } j=j_{\text {rand }} \\
x_{i, j,} \text { otherwise }
\end{array}\right.
$$

$$
\begin{aligned}
& u_{i, j} \\
& =\left\{\begin{array}{c}
x_{i, j}+F \cdot\left(x_{\text {best }, j}-x_{i, j}\right)+F \cdot\left(x_{r 1, j}-x_{r 2, j}\right)+F \cdot\left(x_{r 3, j}-x_{r 4, j}\right), \\
x_{i, j,} \text { otherwise }
\end{array} \quad \text { if rand }[0,1)<C R \text { or } j\right.
\end{aligned}
$$

$$
u_{i, j}=\left\{\begin{array}{c}
x_{r 1, j}+F \cdot\left(x_{r 2, j}-x_{r 3, j}\right)+F \cdot\left(x_{r 4, j}-x_{r 5, j}\right), \quad \text { if } \operatorname{rand}[0,1)<C R \text { or } j=j_{\text {rand }} \\
x_{i, j,} \text { otherwise }
\end{array}\right.
$$

$$
U_{i, G}=X_{i, G}+K \cdot\left(X_{r 1, G}-X_{i, G}\right)+F \cdot\left(X_{r 2, G}-X_{r 3, G}\right)
$$

where $X_{i, G}=x_{i, 1}, x_{i, 2} \ldots, x_{i, j}, j=1,2,3 \ldots D(D=$ dimensionality of the parameter vector $)$ is the $\mathrm{i}^{\text {th }}$ parameter vector in iteration $G ; U_{i, G}=u_{i, 1}, u_{i, 2} \ldots, u_{i, j}$ is the new trial parameter vector; $F$ is a positive control parameter for scaling the difference vectors; $X_{b e s t, G}=x_{b e s t, 1}, x_{b e s t, 2} \ldots, x_{b e s t, j}$ is the parameter vector with the best fitness in the current population and $r 1, r 2, r 3, r 4, r 5$ are mutually exclusive random integers generated within the range $(0, N P)$; and $N P$ is the population size. $C R$ is the crossover probability, rand $[0,1)$ is a random number generated from a uniform distribution between 0 and 1 and $j_{\text {rand }}$ is a randomly chosen integer in the range $(0, D) . K$ is the total number of mutation strategies.

After calculating $U_{i, G}$, it is compared with its counterpart parameter $X_{i, G}$. If the fitness of $U_{i, G}$ is better than $X_{i, G}$, it replaces $X_{i, G}$ in the next iteration. Otherwise, $X_{i, G}$ continues to the next iteration as the new parameter vector.

The algorithm keeps track of the performance of four mutation strategies in the latest $N_{\text {last }}$ iterations to calculate the probability of using each mutation strategy in the next iteration. This 
probability is considered as a performance metric for each mutation strategy. Therefore, the algorithm uses these probabilities to determine how frequently each mutation strategy is to be used in subsequent iterations. Thus, the mutation is self-adapted to the current state of the optimization process. For example, if a certain mutation strategy had a higher probability of generating a successful parameter vector, then that strategy is more frequently used in the next iteration to generate new parameter vectors.

Depending on the number of free parameters, 50,100 or 200 individuals in the parameter population were used. Normally distributed random numbers with mean 0.5 and variance 0.3 for $F$ for each individual, $C R=0.3$ and 0.8 and $N_{\text {last }}=50$ were used for other optimization control parameters, which were obtained from the original study of SaDE by Qin et al. (25). The optimization was terminated if the fitness was not improved more than a tolerance value $\left(1 \mathrm{e}^{-4}\right.$ or $\left.1 \mathrm{e}^{-6}\right)$ over 1000 iterations (stopping criteria).

\subsection{Generating stochastic stimuli}

We tested white, synaptic and pink noise stimuli for their impact on modeling performance. White noise input was generated by utilizing the standard normrnd function in Matlab (R2014b, Natick, MA). The synaptic noise model was developed based on the study of Destexhe et al. (26), and pink noise signal was generated by the code provided by Zhivomirov (27).

\subsection{Cell preparation and electrophysiology}

CHO cells were cultured in Dulbeccos Modified Eagle Medium: Nutrient Mixture F-12 (Thermo Fisher Scientific, Waltham, MA) supplemented with 10\% (v/v) fetal bovine serum (Thermo Fisher Scientific) and $50 \mathrm{IU} \mathrm{ml}^{-1}$ penicillin (Thermo Fisher Scientific) at $37^{\circ} \mathrm{C}$ with $5 \% \mathrm{CO}_{2}$ in $75 \mathrm{~cm}^{2}$ flasks. At $\approx 80 \%$ density, CHO cells were transiently co-transfected with human R1882Q Nav 1.2 channel variant (4 g) and enhanced green fluorescent protein (1 g) using Lipofectamine 3000 Reagent (Thermo Fisher Scientific). The R1882Q Nav 1.2 channel was used because in our previous DAPC study this variant resulted in higher AP firing compared with wild-type (28). 24 hours posttransfection, the cells were plated on $13 \mathrm{~mm}$ diameter glass coverslips (Menzel-Glser, Thermo Fisher Scientific); and electrophysiological recording were performed 48-72 hours post-transfection. Wholecell patch clamping was performed using boroscilicate glass pipettes pulled on a horizontal puller (P97, Sutter Instruments) to resistance values of 1-2 $\mathrm{M} \Omega$ and, filled with a solution containing $5 \mathrm{mM}$ CsCl, $120 \mathrm{mM}$ CsF, $10 \mathrm{mM} \mathrm{NaCl}, 11 \mathrm{mM}$ EGTA, $1 \mathrm{mM} \mathrm{CaCl}, 1 \mathrm{mM} \mathrm{MgCl} 2,2 \mathrm{mM} \mathrm{Na} 2 \mathrm{ATP}, 10$ $\mathrm{mM}$ HEPS ( $\mathrm{pH}=7.3$ with $\mathrm{CsOH})$. Cells were bathed in a solution containing $145 \mathrm{mM} \mathrm{NaCl}, 5 \mathrm{mM}$ $\mathrm{CsCl}, 2 \mathrm{mM} \mathrm{CaCl}_{2}, 1 \mathrm{mM} \mathrm{MgCl}$, $5 \mathrm{mM}$ glucose, $5 \mathrm{mM}$ sucrose, $10 \mathrm{mM}$ Hepes ( $\mathrm{pH}=7.4$ with $\mathrm{NaOH}$ ). 
Currents were recorded at room temperature $\left(22-23^{\circ} \mathrm{C}\right.$ ) using an Axopatch 200B amplifier (Molecular Devices, Sunnyvale, CA) controlled by a Clampex 9.2/DigiData 1440 acquisition system (Molecular Devices). Currents and potentials were low-pass filtered at $10 \mathrm{kHz}$ and digitized at $50 \mathrm{kHz}$. Slow and fast capacitances were compensated and leak currents were subtracted using the amplifiers linear leak subtraction function. Series resistance values, typically of $2.5 M \Omega$, were 90-95\% compensated. Experiments were not included when the calculated series resistance error exceeded $5 \mathrm{mV}$.

\subsection{DAPC implementation}

AP data were obtained in dynamic action potential clamp experiments (19). Heterologously expressed ion channel currents in $\mathrm{CHO}$ cells were incorporated into a biophysically realistic computational model of the distal axon initial segment (AIS) compartment of a cortical pyramidal neuron $(2,28)$. The computational model contained an active fast rectifying potassium current $\left(I_{K}\right)$ and passive leak current $\left(I_{\text {leak }}\right)$ and membrane capacitance $\left(C_{m}\right)$. The model was implemented in Simulink (Mathworks, Natick, MA) and converted to a $\mathrm{C}$ library using Matlab real-time workshop. The $\mathrm{C}$ library was then run by an ADwin Pro II real-time processing unit (Jäger Computergesteuerte Messtechnik GmbH, Lorsch,Germany) equipped with a Tiger Sharc ADSP-TS101S processor from Analog Devices (Norwood, MA, USA) with $300 \mathrm{MHz}$ clock rate, $768 \mathrm{kB}$ local memory and 256 MB RAM. The CHO cell expressing $\mathrm{Na}_{\mathrm{v}} 1.2$ channels was coupled with the model via the Axopatch 200B amplifier. The amplifier was connected to the ADwin processor through ADwin analog input card. The processor enables computationally intensive real-time computing with precision and high speed of $140 \mathrm{kHz}$ (time-step: $\mathrm{dt}=7.14 \mu \mathrm{s}$ ) using the explicit Euler method. The explicit Euler method was selected over implicit methods (Backward Euler) or combined methods (Crank-Nicholson) as the discrete solver due to its ease of implementation and speed compared to the other two solvers (29). Synaptic current $\left(I_{s y n}\right)$ was used as stimulus to evoke APs in the 'hybrid' neuron model. At every iteration step, the CHO cell's current was recorded and scaled by a scale factor to keep inward peak sodium channel current constant throughout different experiments. As mentioned earlier, this scaled current was fed into the computational model using the command potential function of the amplifier to generate a virtual current clamp mode recording. The corresponding membrane potential $\left(V_{m}\right)$ calculated by the model was fed back to the CHO cell through the amplifier as the command potential $\left(V_{c m d}\right)$. In all DAPC experiments, the non-scaled and scaled cell current input, the stimulus waveform, $V_{m}$ and the total synaptic current in the model cell were simultaneously recorded. The various conductances in the AIS compartment, $C_{m}$ and synaptic current amplitude and the scaling factor for $\mathrm{CHO}$ cell current were adjusted simultaneously during the experiments enabling immediate intervention if required for quality control purposes. The files were stored as Axon Binary Files (.abf) and later converted to .mat files for further analysis. 


\subsection{Verifying the modelling approach}

To verify the modelling approach, two simulated datasets from the Type 1 model (with and without external noise) and four experimental datasets were used. Simulated datasets were used to compare the performance of VC and DAPC based methods (Section 3.1), to identify an optimal stimulation method for DAPC (Section 3.2) and to evaluate the two-step optimization with noisy DAPC data (Section 3.3.1). The verifications were performed using a similar approach to those adopted in $(10,30)$. In fully-simulated DAPC mode, the sodium conductance corresponds to an in silico sodium channel model described in Section 2.1, whereas the computation model incorporates an active potassium current $\left(\mathrm{I}_{\mathrm{K}}\right)$, a passive leak current $\left(\mathrm{I}_{\text {leak }}\right)$ and membrane capacitance $\left(\mathrm{C}_{\mathrm{m}}\right)$, as described in Section 2.1. Datasets from real DAPC experiments were used to verify the DAPC modelling approach and to calculate the predictability of this approach in real world tests (Section 3.3.2). In real DAPC mode, we implemented heterologously expressed $\mathrm{Na}_{\mathrm{v}} 1.2$ conductance as described in Section 2.7.

The optimization algorithm was implemented in Matlab (R2014b, Natick, MA) and was run in a desktop computer with core i7 $3.60 \mathrm{GHz}$ processor and $16 \mathrm{~GB}$ memory running Windows 7 . The fitness function calculation for each parameter set was performed in parallel using the Matlab Parallel Computing Toolbox to reduce computation time. The Source code for the DAPC implementation and the optimization algorithm are available as supplementary material. 


\section{RESULTS}

\subsection{DAPC analysis improves parameterization accuracy for a sodium conductance model}

There are two major approaches for extracting parameters from an ion channel conductance. Voltage clamp (VC) is the most common method to generate current data that can be fit with $\mathrm{HH}$ formalisms. The DAPC method generates membrane current data with additional dynamic voltage data, however its utility in parameterizing $\mathrm{HH}$ formalisms has been less well explored. Here, we compare VC-derived data fitting methods with DAPC data fitting methods to formally establish the relative strengths and weaknesses of these two approaches.

We simulated VC and DAPC experiments to compare their accuracy in parameterizing conductance models. In the virtual VC experiment, we determined reference conductances during activation and steady-state fast inactivation using a Type 1 sodium channel model (see Section 2.1) (Fig. 2a). Virtual DAPC experiments were performed using a single compartment model incorporating the Type 1 sodium channel model together with the Type 1 potassium channel model and leak conductance. AP firing was elicited by a $20 \mu \mathrm{Acm}^{-2}$ step current of $200 \mathrm{~ms}$ duration (Fig. $2 \mathrm{~b}$ ). Data from these VC and DAPC experiments were fit with the standard HH model equations (see Section 2.1) to estimate the parameter values. Both standard and improved VC fitting methods (see Section 2.2) were employed for parameterizing the VC data. As shown in Fig 2c, representative conductance traces generated using parameters obtained from the standard VC fitting method deviated significantly from the reference traces (Fig 2a, left). As predicted from and confirming earlier work (1), the conductance based on parameters obtained with the improved VC fitting method matched the reference trace more closely than with the standard method. The complete activation and inactivation conductance traces generated from the fitted models from both VC methods are shown in Fig. S1. The validity of the DAPC approach was confirmed by the finding that the membrane potential traces generated from the model of proposed DAPC fitting method faithfully recapitulated the time course and shape of the reference trace (Fig 2d).

The data generated by both VC and DAPC allowed accurate estimates of the $\mathrm{HH} m_{\infty}$ and $h_{\infty}$ parameters (Fig 3a). However, using the standard VC fitting technique the activation and inactivation time constants ( $\tau_{m}$ and $\tau_{h}$, respectively) deviated significantly from the reference values, whereas the improved fitting method showed very good agreement with $\tau_{m}$ and a small deviation for $\tau_{h}$ that increased at more negative potentials (Fig 3b). In contrast, the estimates of $\tau_{m}$ and $\tau_{h}$ obtained from analysis of the DAPC data resulting in a significant improvement for both time constants that was consistent over the voltage range shown (Fig 3b). Overall, the error difference was more pronounced 
using standard VC fitting method for the parameters related to the time constants (Fig 3c: $a_{m}, b_{m}, a_{h}, b_{h}$ ), which were all >30\% (Fig 3c). Moreover, the standard VC method sets maximum conductance $\left(G_{N a}\right)$ based on the measured maximum value in activation conductance curves, whereas both the improved VC method and DAPC approach treat $G_{N a}$ as free parameter. Therefore, the $G_{N a}$ error was low for these two methods compared with the standard VC model (Fig. 3c). Further corroborating these results, the APs predicted by the fitted model from DAPC overlapped with the reference APs while the model from improved VC method predicted APs with a small time shift for the second AP. However, the fitted model from standard VC failed to predict the second AP (Fig. 3d). Taken together, these findings established that parameterization of the HH model using the DAPC approach yielded a significant improvement in accuracy compared with the VC approach, even when employing the improved fitting method. 
a)

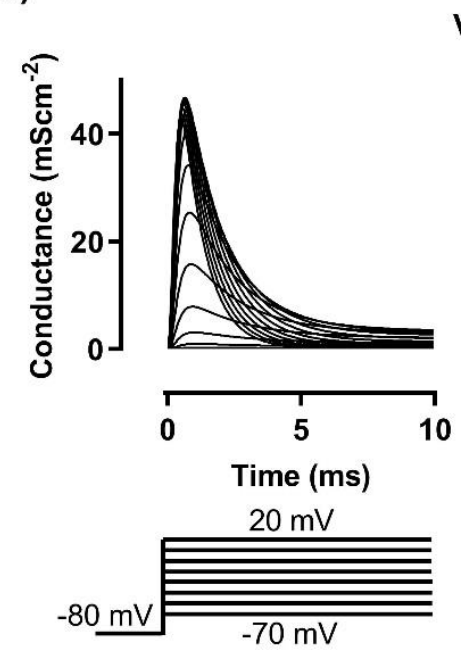

VC

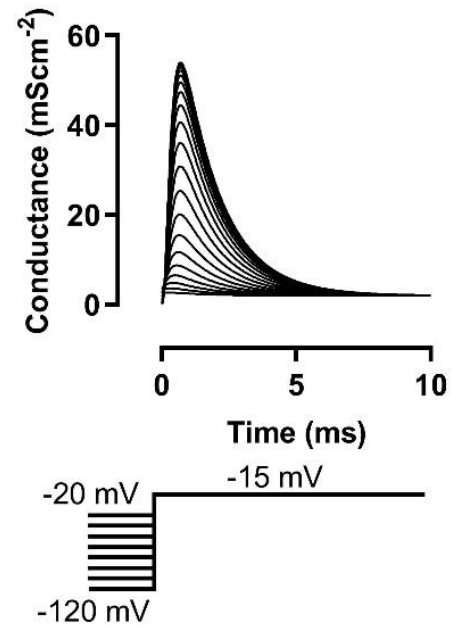

b)

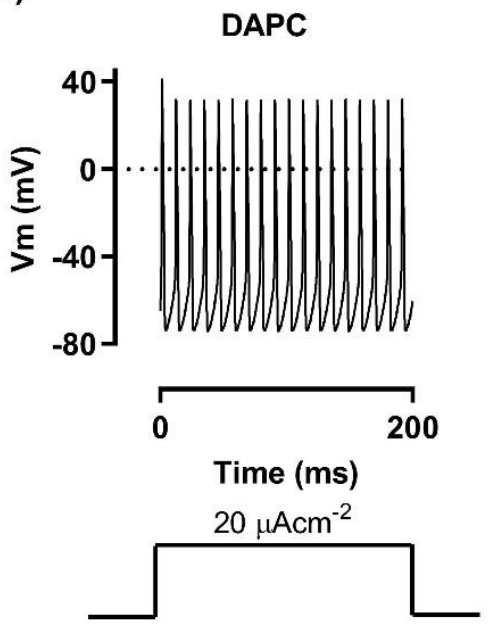

c)
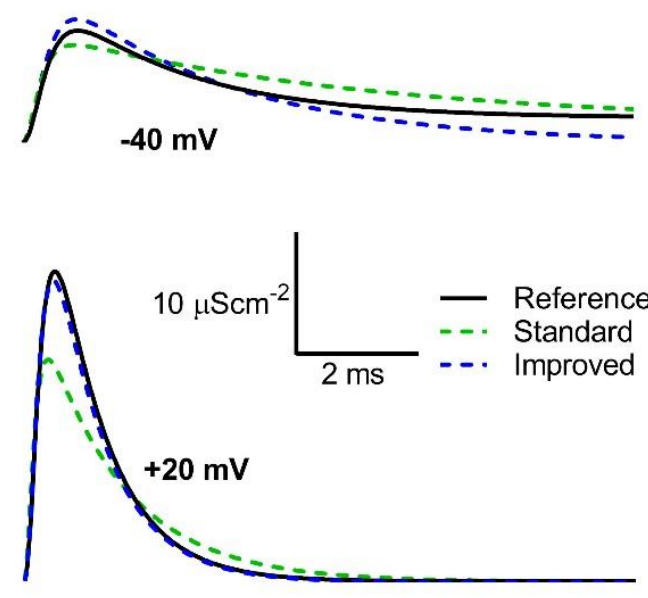

d)
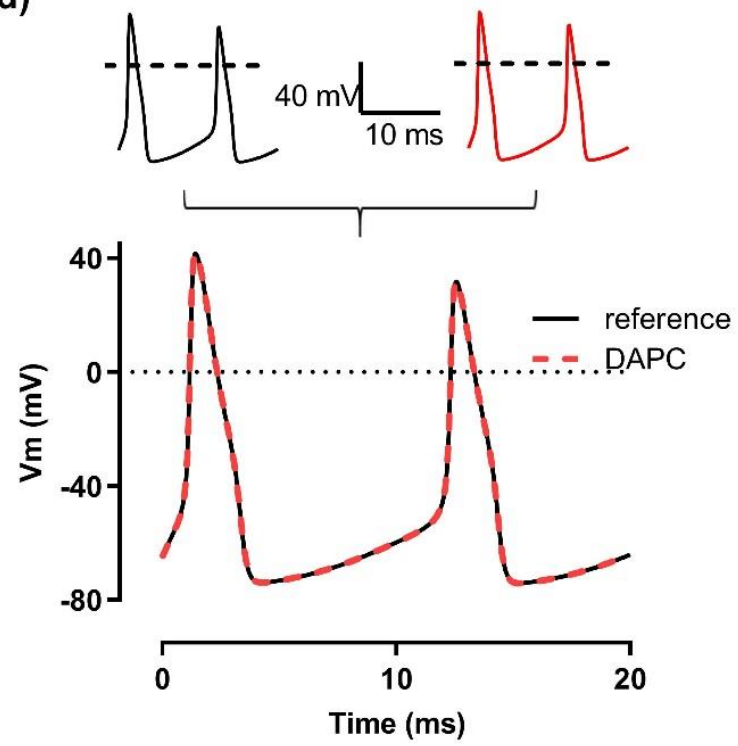

Figure 2. Parameterizing conductance models in simulated VC and simulated DAPC experiments. (a) VC simulations for activation conductance traces (left panel) and inactivation (right panel) according to the voltage protocols shown below each data set. (b) APs elicited by $20 \mu \mathrm{Acm}^{-2}$ step current (constant stimulation) in simulated DAPC. (c) Simulated VC activation conductance curves for two test voltage steps $(-40 \mathrm{mV},+20 \mathrm{mV})$ taken from a) compared with the corresponding curves derived from the fit parameters estimated from the standard VC fitting method (blue dashed lines) and improved VC fitting method (green dashed lines) (Eqs. 7-10). (d) Comparison of APs reconstructed from the fitted model (red dashed traces) and simulated DAPC APs (black) from b) for a $20 \mathrm{~ms}$ interval plotted separately (upper traces) and overlapped (lower traces). Dashed lines indicate zero membrane potential. 
a)

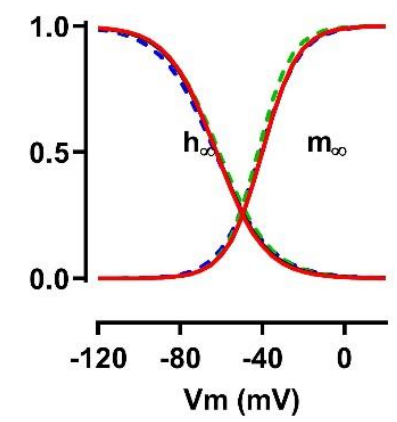

c)

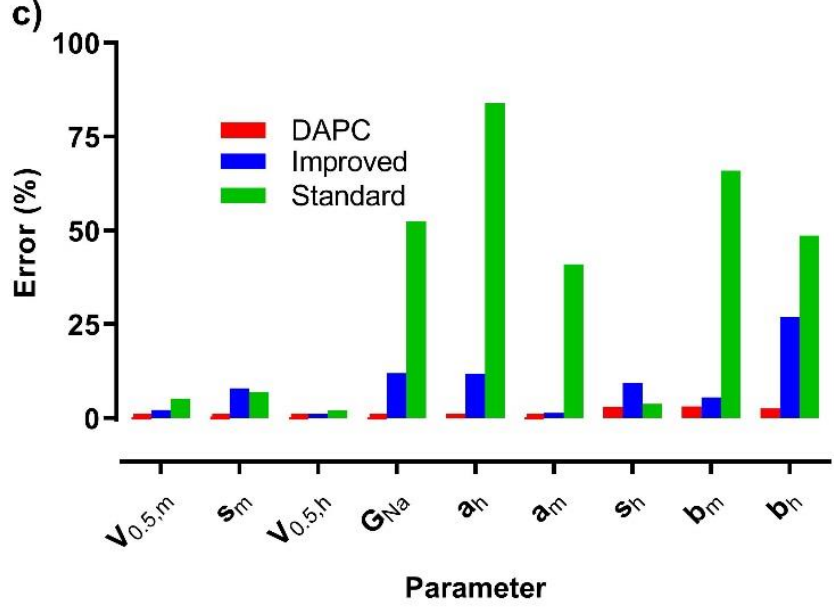

b)

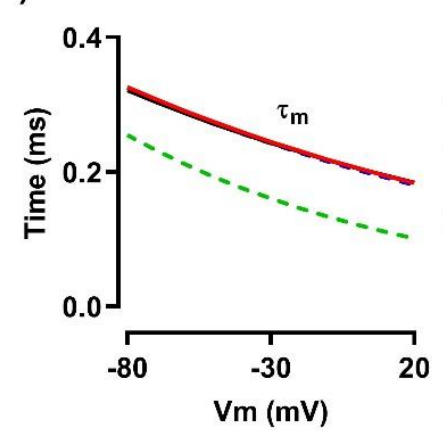

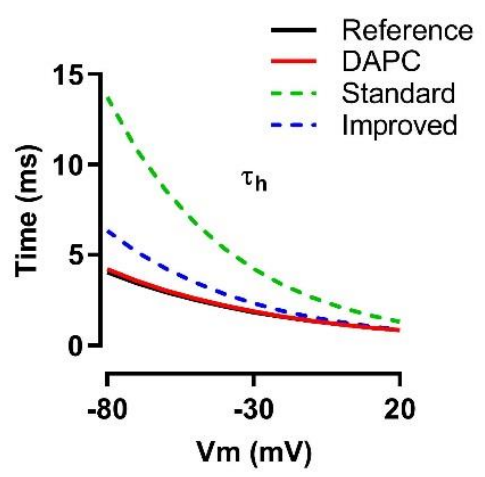

d)

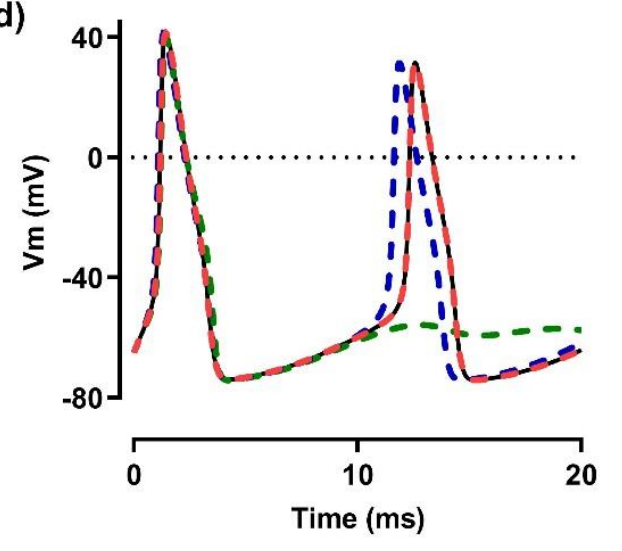

Figure 3. Quality of sodium channel parameters for the HH type model based on DAPC and VC data. (a) Voltage dependence of steady-state activation $\left(m_{\infty}\right)$ and inactivation $\left(h_{\infty}\right)$. (b) Activation $\left(\tau_{m}\right)$ and inactivation $\left(\tau_{h}\right)$ time constants. In (a) and (b) curves were derived from fitted models of standard VC (blue dashed lines), improved VC fitting methods (green dashed lines), and DAPC (red solid lines), and were compared with corresponding reference curves (black solid lines). (c) Mean error, expressed as a percentage of reference parameters, resulting from standard VC (blue), improved VC (green) and DAPC (red) fitting methods. Error bars were not shown as they were $<1 \%$ in all cases. Parameters are ordered with increasing sensitivity from left to right on the $\mathrm{x}$-axis. Sensitivity is defined as the change in AP firing rate for a constant stimulus in the model neuron when parameters are shifted (see Fig. S2). (d) Comparison of APs predicted from the fitted models of standard VC (blue dashed lines), improved VC (green dashed lines), and DAPC (red dashed lines) fitting methods, and were compared with corresponding reference curves (black solid lines). Dashed lines indicate zero membrane potential. 


\subsection{Synaptic noise identified as optimal stimulation strategy}

To further optimize the use of DAPC in parameterizing $\mathrm{HH}$ formalisms, we next investigated possible input stimulation strategies. A standard constant current step protocol $(11,12,31)$ and a more biologically realistic synaptic noise stimulating protocol (31) of identical duration were investigated and their impact on identifying reference parameters was compared(Fig 4). Step stimuli were simulated as current sources, whereas synaptic noise was simulated as combinations of time varying excitatory $\left(G_{e}\right)$ and inhibitory $\left(G_{i}\right)$ conductances based on the Ornstein-Uhlenbeck process (26). The amplitudes and reversal potentials of currents, and the amplitudes and variances of conductance were selected from earlier work (26) and modified to ensure that the output voltage traces had approximately the same total power in the output spectrum. These stimuli were applied to a fully simulated DAPC system comprising a single compartment model incorporating the Type 1 sodium channel model together with the Type 1 potassium channel model and leak conductance (Figs. 4a and 4b). This fully simulated DAPC system was run for $200 \mathrm{~ms}$. The generated membrane potential data were fed to the optimization algorithm with population size of $50, \mathrm{CR}=0.3$ using fitness function $\psi_{1}$. Nine free parameters were optimized, based on the HH formalism of our model cell. The optimization was repeated 20 times for each stimulation method, but with different initial populations drawn randomly from a uniform distribution over the parameter space. Figure S6 shows the evolution of model accuracy with iterations in the optimization algorithm for one such optimization instance. Both stimulation strategies resulted in membrane potential traces overlapping well with reference (Figs. $4 a$ and $4 b$ ), suggesting that the quality of the underlying fits was similar. As shown in Fig $4 c$, the estimated parameter errors were less than $5 \%$ for both stimulation strategies.

Next, we investigated the impact of individual stimulation strategies on modeling performance by measuring the computational complexity in terms of the number of iterations required for convergence of parameter estimation. The step stimulation strategy yielded data sets that on average converged within 5,000 iterations. Analysis of the computational efficiency for modeling data generated by synaptic noise stimuli was clearly superior over the step simulation strategy as it typically converged within 2,500 iterations (Table S1). The average computational time required for the modeling was approximately 18 minutes for synaptic noise compared with 40 minutes for constant current step (running a PC with an i7 $3.60 \mathrm{GHz}$ core processor and $16 \mathrm{~GB}$ memory under Windows 7). We also investigated the modeling performance with less commonly used stimulation strategies such as a staircase current (32-34), white noise (12), pink noise (16) (Figs. S3 and S4). This extended study showed that the performance characteristics of pink noise and synaptic noise were similar, nevertheless, for the remainder of the study we used Ornstein-Uhlenbeck synaptic noise stimulation as it has been better characterized for neuronal modeling and represents a physiologically more realistic stimulus (26). 
Finally, we also explored the utility of DAPC-based modeling and computational complexity for a potassium channel conductance. We implemented a virtual DAPC experiment comprising a single compartment model incorporating the Type 1 potassium channel model together with the Type 1 sodium channel model and leak conductance. This virtual DAPC configuration was stimulated using synaptic noise for a period of $200 \mathrm{~ms}$ and membrane potential was acquired. To extract the original Type 1 potassium channel reference parameters, the optimization was run 20 times, each with different initial populations randomly generated within the parameter space. For parameterization, $365 \pm 12$ iterations were needed for convergence and the estimated values were significantly more accurate than those estimated for sodium channels, resulting in an average error $<0.04 \%$ (Fig S5). The average realtime required for optimization was around 3 minutes when using the same PC as above. This performance increase is presumably due to the reduced complexity of the conductance models used for the potassium channel compared to sodium channel. Taken together, these results demonstrate that this approach can generalize to other types of channels. 
a)
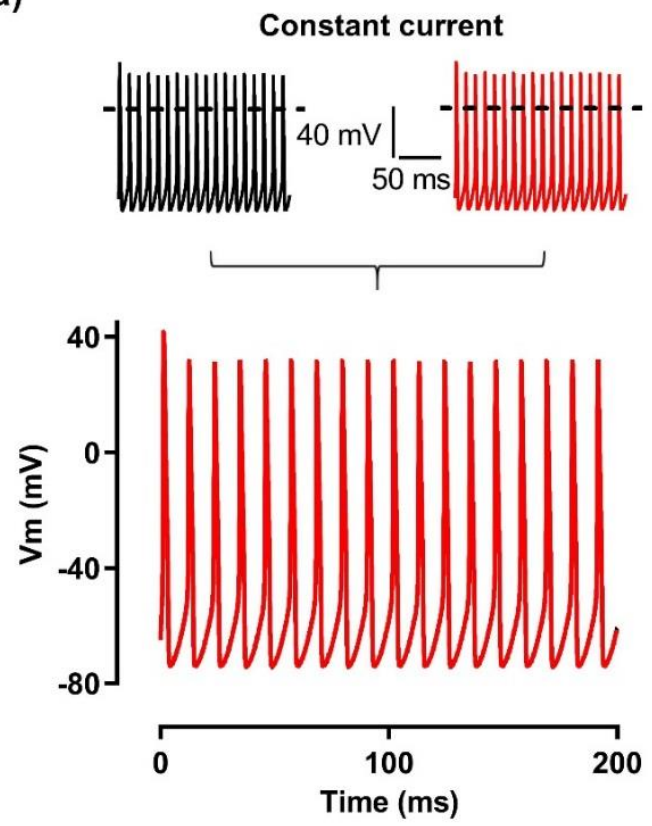

b)
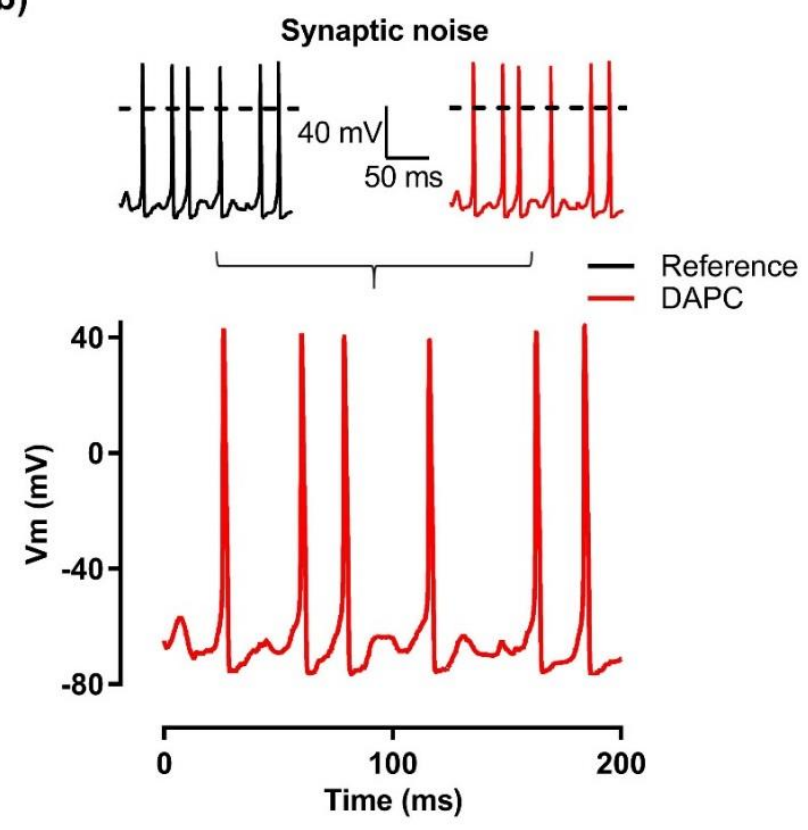

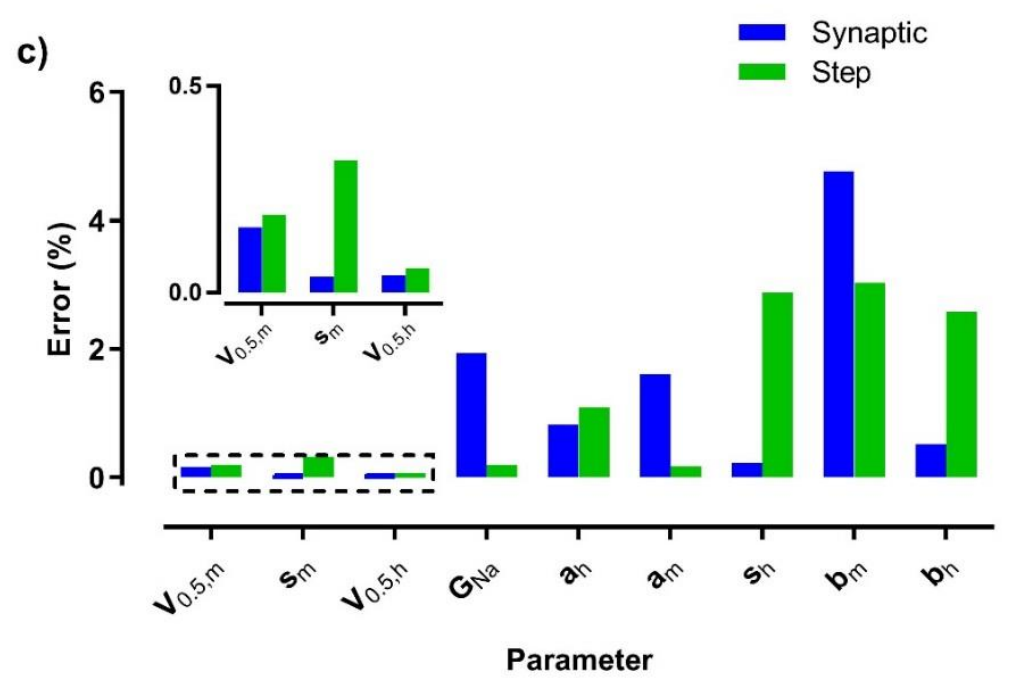

Figure 4. Performance of the modeling approach based on DAPC data acquired from step and synaptic noise stimulations. Reference voltage traces of 200 ms duration, acquired upon stimulating the DAPC with constant (a) current step and (b) synaptic noise (black) and corresponding traces from fitted models (red) plotted separately (top) and overlapped (bottom). Dashed lines indicate zero membrane potential. (c) Effect of step and synaptic noise stimuli on the average parameter error during the optimization process (blue: synaptic noise; green: constant current). Error bars were not shown as they were $<1 \%$ in all cases. Note that parameters are ordered with increasing sensitivity from left to right on the $\mathrm{x}$-axis. The inset shows the parameter error for $V_{0.5, m}, s_{m}$ and $V_{0.5, h}$ (boxed area) enlarged for clarity. 


\subsection{Estimating heterologously expressed $\mathrm{Na}_{v}$ conductance parameters using DAPC}

To evaluate the performance of DAPC based conductance parametrization workflow (Fig. 1c), we characterized the voltage responses of a hybrid cell model incorporating voltage-gated Na current expressed in CHO cells, and leak and potassium currents represented solely in the in-silico model (28). These experiments are typically influenced by external noise as discussed below. In typical DAPC recordings, we estimated a SNR of $52 \pm 1.26 \mathrm{~dB}$ ( $\mathrm{n}=8$ cells). Hence, we assessed and adjusted our optimization approach to become robust against external noise and we applied this improved optimization approach to parameterize a conductance model of $\mathrm{Na}_{\mathrm{v}}$ variant channels.

Although a variety of signal processing techniques can be used to minimize unwanted noise in an electrical system, it is impossible to remove noise completely. Two types of noise contribute to the overall DAPC noise.: 1) intrinsic cell noise of the biological system, arising from a combination of current fluctuations due to channel openings and closing, ion permeation and capacitance noise due to cell membrane properties, which cannot be reduced; and 2) extrinsic instrumentation noise, which can be a major source of noise in typical experiments. The impact of this noise can be minimized with good practice such as shielding, coating and material properties of recording electrodes and selection of headstage electronics. In a DAPC system, the signal-to-noise ratio (SNR) of the current or voltage output is the ultimate determinant of the performance of the feedback loop and consequently of the accuracy of parameter estimation.

\subsubsection{Two step optimization improves the parameterization efficiency of DAPC method}

To better understand the impact of noise on the accuracy of parameter estimation, we included a Gaussian noise source in a simulated DAPC experiment (Fig 5a) and the effect of SNR on DAPC performance was evaluated by measuring the AP firing frequency difference between simulations with and without noise over a 10 s period. Fifty DAPC experiments were simulated for each SNR value, each with a unique Gaussian noise sequence. The experiment was repeated without a stimulus and using a current step stimulus input of three times the rheobase. The curves in Fig $5 b$ and $5 c$ show the average and standard deviations of the frequency shift and SNR for each of these experiments. As seen in Fig $5 b$ and $5 c$, the frequency shift has notably increased for SNR $<50 \mathrm{~dB}$.

To analyze the performance of the modeling approach, a simulated noisy DAPC experiment with $\mathrm{SNR}=52 \mathrm{~dB}$ (mean SNR identified from the experimental recordings above) was utilized. The effect of external noise for neuron model parameter estimation was evident for $\mathrm{SNR}=52 \mathrm{~dB}$, as shown in Fig 5d. As expected, the estimated parameter accuracy was reduced when noisy traces were used for modeling. The averaged parameter error based on non-noisy traces was $\approx 1.16 \%$ whereas it was $\approx$ 
$62 \%$ when noisy trace was utilized, with parameters $S_{h}$ and $b_{m}$ exhibiting errors $\geq 100 \%$ when modeling with the standard one-step method. The effect of noise can be less in APs compared to subthreshold regime due to high signal power when firing. Therefore, to improve the performance of parameter estimation, a second step was introduced after fixed number of iterations in the one-step method, which enabled optimization based on fitness function $\psi_{2}$ (see Section 2.4). This improved method is referred to as the two-step method hereafter. Although the parameter errors were higher compared to the estimation based on non-noisy trace, the two-step optimization method could improve the accuracy of modeling for SNR of $52 \mathrm{~dB}$. The averaged estimation error of parameters for two-step method was $\approx 8 \%$, with $s_{h}$ being an outlier having an average error of $47 \%$. The error in $s_{h}$ is acceptable as the sensitivity of the neuron firing rate to $s_{h}$ is very low. For example, the AP firing rate of the model does not change by more than $4 \%$ when stimulated by step currents, even if $s_{h}$ is increased by $50 \%$ (Fig S2). Therefore, the two-step optimization method is a better choice with noisy data for accurate parameter estimation. 
a)

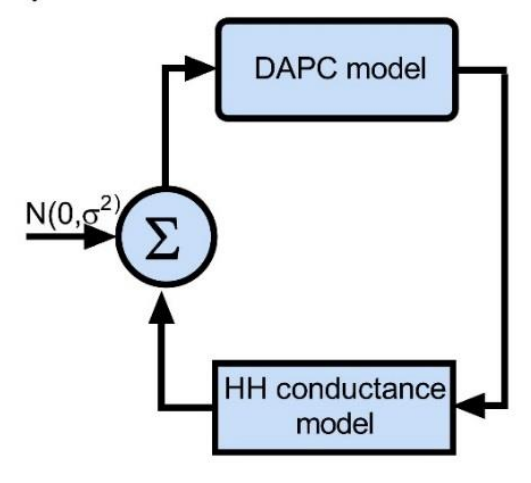

b)

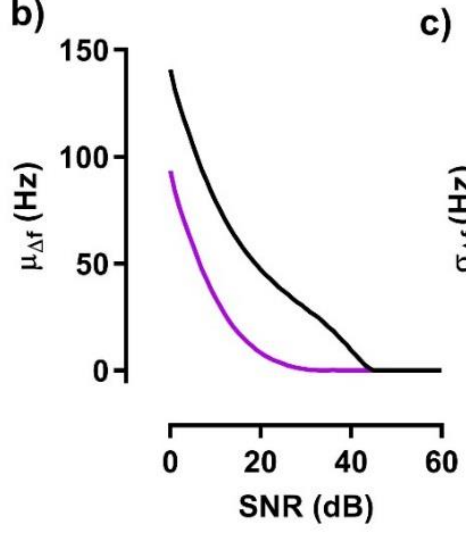

c)

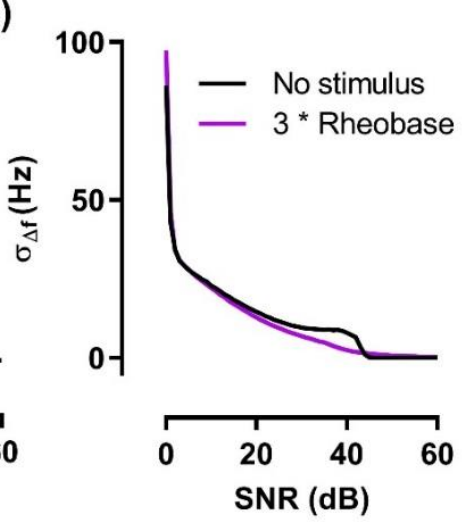

d)

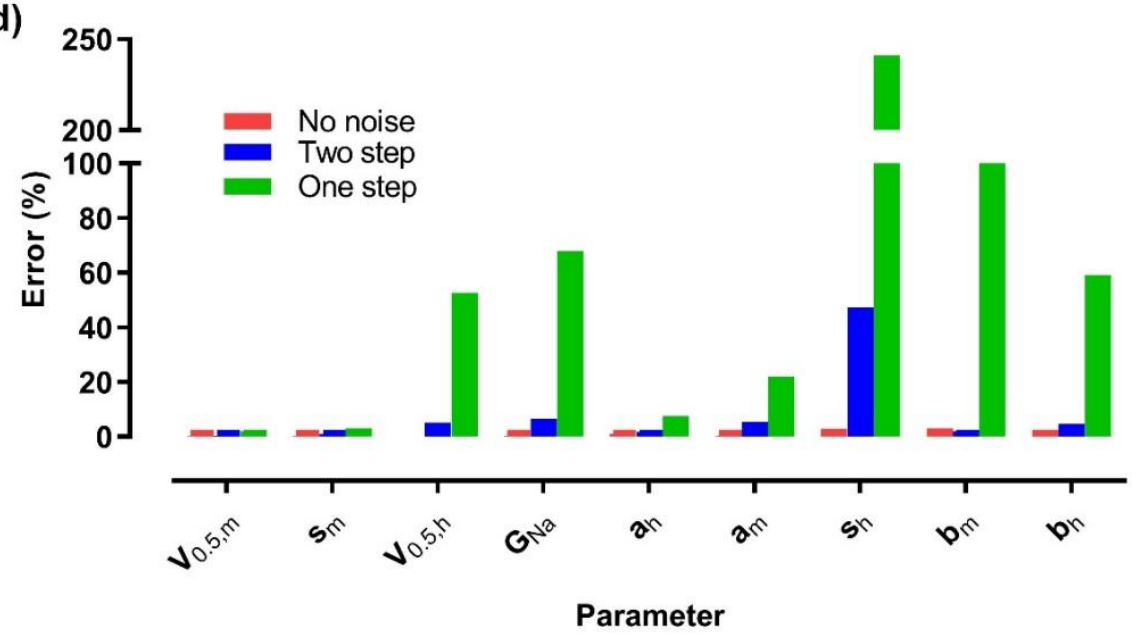

Figure 5. A two-step process improves neuron modeling based on noisy DAPC data. (a) Simulating a noisy DAPC system with a Gaussian noise signal added to the simulated ion channel current. The signalto-noise ratio (SNR) was varied from 0 to $60 \mathrm{~dB}$ in $1 \mathrm{~dB}$ increments, and a $10 \mathrm{~s}$ membrane potential trace was acquired to measure the effect of noise on AP firing frequencies. The simulation was repeated 50 times for each SNR by varying the Gaussian noise signal. (b) and (c) Mean and standard deviation of the AP firing frequency shift, respectively, relative to the frequency of a non-noisy trace. Note that for SNR $<50 \mathrm{~dB}$, the frequency shift increased significantly (black line: frequency shift at rest (No stimulus); purple line: step stimuli of three times the rheobase current). (d) Mean error of estimated parameters based on non-noisy trace (red) and noisy traces using standard one-step method (green) and proposed two-step method (blue). Error bars were not shown as they were $<1 \%$ in all cases. The parameter errors were significantly increased due to external noise; however, the two-step method could reduce this discrepancy. Parameters are ordered with increasing sensitivity from left to right on the $\mathrm{x}$ axis. 


\subsubsection{Kinetic modeling using real DAPC data of transiently expressed human Nav1.2 ion channels}

Sodium current recorded form $\mathrm{CHO}$ cells heterologously expressing $\mathrm{Na}_{\mathrm{v}} 1.2$ channels was incorporated into a biophysically realistic neuronal compartment model comprising in silico potassium (Type 2) and leak conductances, thus establishing DAPC configuration. AP firing was elicited by using the scalable model of synaptic noise similar to the simulated experiments above.

Parameterizations were undertaken using datasets that included a minimum of four APs and typically ranged from 400-1200 ms. We performed kinetic modeling in a series of independent experiments $(n \geq 4)$. Figure 6 a shows membrane potential changes and corresponding sodium currents (Fig. 6b) from one experiment where $1000 \mathrm{~ms}$ of DAPC recording time was used to produce a $\mathrm{HH}$ model of the expressed voltage-gated sodium conductance using the two-step optimization method that could accurately reproduce the firing and current response of the recorded data. The estimated parameters of this model are given in Table 1. As a further test of the performance of this modeling approach, the ability of the model to predict the firing of the cell in DAPC mode was tested in periods outside of the epoch used for parametrization. In the representative example shown in Fig 6c, the model corresponded well to a $4 \mathrm{~s}$ period following the optimization epoch, resulting in a firing accuracy of $95 \%$ and an overlapping accuracy of $100 \%$. Average data from four independent experiments resulted in $96 \% \pm 3 \%$ in firing rate accuracy and a $94 \% \pm 2 \%$ temporal overlap accuracy. The analysis of AP morphology and $\mathrm{I}_{\mathrm{Nav1.2}}$ time course of reference versus fitted data also showed a very high level of correspondence (Figs. 6a and 6b, respectively), further validating the accuracy of the modeling approach. Overall the DAPC modeling approach was capable of predicting not only the AP firing rate but also the timing and AP shape with high accuracy and could be applied to parameterize various neuronal conductances. 
a)

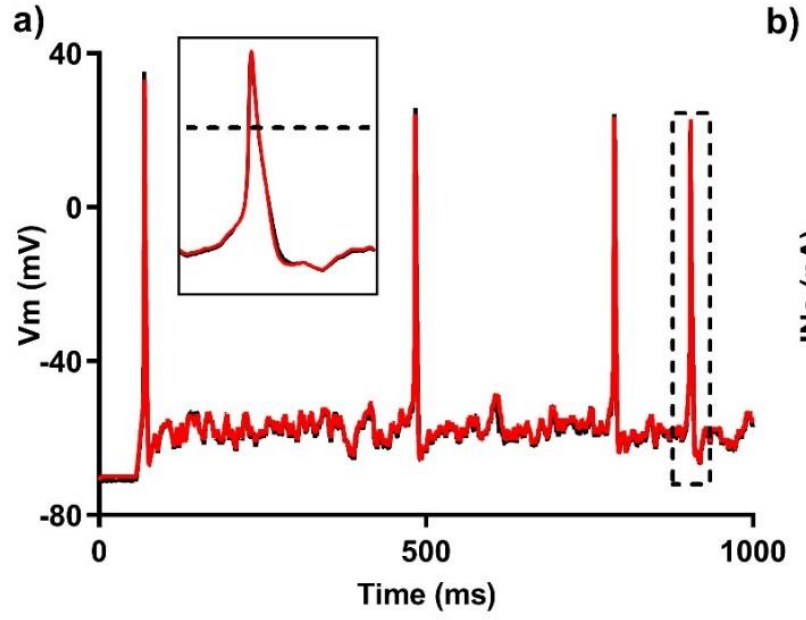

b)

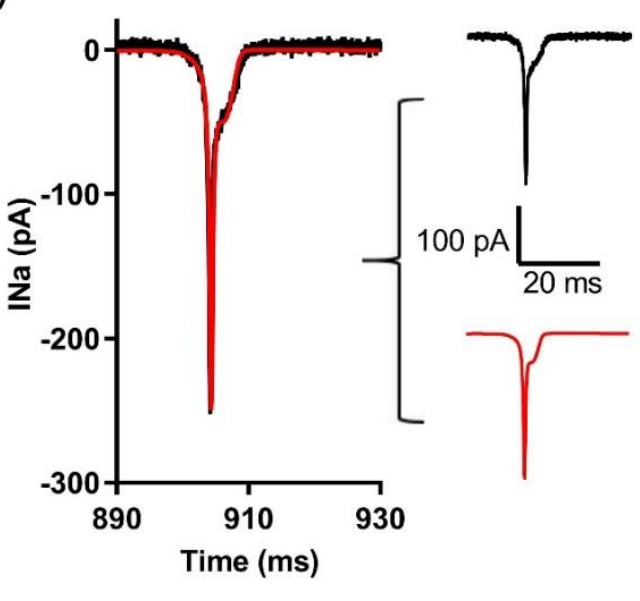

c)

- Experimental
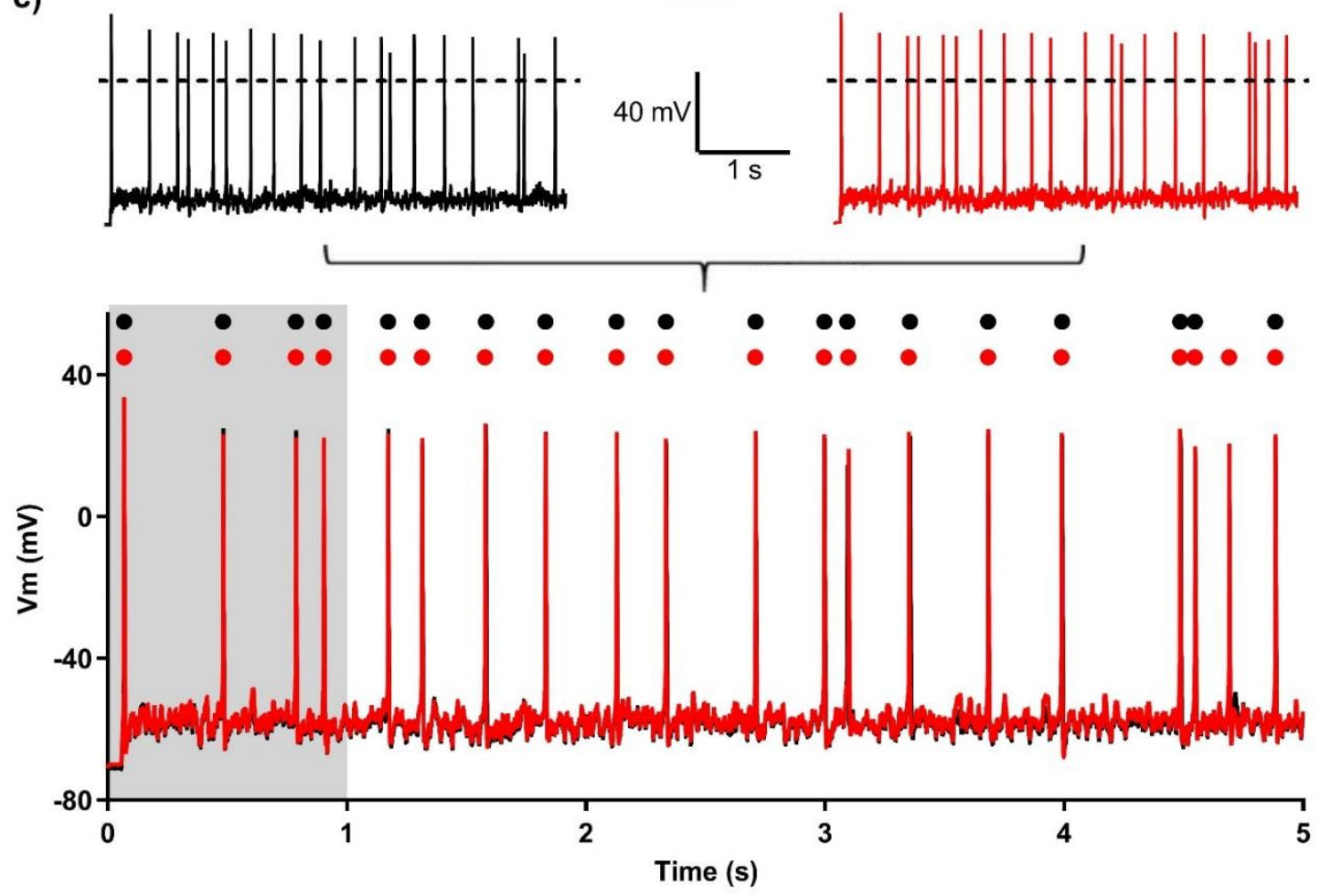

Figure 6. Kinetic modeling using real DAPC data from the "hybrid" neuron comprising the in silico model cell and the $\mathrm{CHO}$ cell expressing $\mathrm{Na}_{\mathrm{v}} 1.2$. (a) The DAPC system was stimulated by synaptic noise resulting in dynamic $\mathrm{V}_{\mathrm{m}}$ changes. In this experiment, one second of the recorded membrane potential (black: reference trace) was used to estimate the parameters of a $\mathrm{HH}$ type model for $\mathrm{Na}_{\mathrm{v}} 1.2$ channels. The $\mathrm{V}_{\mathrm{m}}$ trace generated from the model (red line) closely followed the reference (black). The inset shows an enlarged AP; the zero $\mathrm{V}_{\mathrm{m}}$ is indicated by the dotted line. (b) Overlapped $\mathrm{I}_{\mathrm{Nav1.2}}$ traces generated from the model (red, right inset) or recorded in the DAPC experiment (reference, black, right inset) during an AP. Note the absence of the high frequency fluctuations in the sub-threshold modeled current data compared with real experimental data. (c) Correspondence of the modeled (red trace, top inset) 
and the experimental traces (black, top inset). The first $1 \mathrm{~s}$ of experimental data was used to model the sodium conductance (shaded area), whereas the subsequent $4 \mathrm{~s}$ was used to predict model behavior compared with experimental data. The model could predict $4 \mathrm{~s} \mathrm{~V}_{\mathrm{m}}$ changes with $100 \%$ AP overlapping accuracy, whereas the average firing rate accuracy was $95 \%$. The red- and black-filled circles above the $\mathrm{V}_{\mathrm{m}}$ traces indicate the presence of experimentally recorded (black) and modeled (red) APs. 
Table 1: Parameters of the HH model during real cell DAPC experiments.

\begin{tabular}{cccccc}
\hline Parameter & R1882Q Na 1.2 & Parameter & R1882Q Nav 1.2 & Parameter & R1882Q Na 1.2 \\
\hline$S_{m}$ & $-0.15 \pm 0.03 \mathrm{mV}^{-1}$ & $b_{m}$ & $0.04 \pm 0.01 \mathrm{mV}^{-1}$ & $\tau^{0}$ & $0.40 \pm 0.12 \mathrm{~ms}$ \\
$V_{0.5, m}$ & $-34.59 \pm 1.48 \mathrm{mV}$ & baseh & $0.004 \pm 0.003$ & $a_{h}$ & $0.41 \pm 0.19 \mathrm{~ms}$ \\
$\tau^{0}$ & $0.18 \pm 0.15 \mathrm{~ms}$ & $S_{h}$ & $0.20 \pm 0.04 \mathrm{mV}^{-1}$ & $b_{h}$ & $0.083 \pm 0.02 \mathrm{mV}^{-1}$ \\
$a_{m}$ & $0.15 \pm 0.12 \mathrm{~ms}$ & $V_{0.5, h}$ & $-56.20 \pm 2.54 \mathrm{mV}$ & & \\
\hline
\end{tabular}

The parameters $S_{m}$ (Slope of Steady state activation), $V_{0.5, m}$ (activation half voltage), $\tau^{0} m, a_{m}, b_{m}$ (Coefficients of activation time constant), base $_{h}$ (minimum inactivation) $s_{h}$ (Slope of Steady state inactivation), $V_{0.5, h}$ (Inactivation half voltage), $\tau^{0} m, a_{h}, b_{h}$ (Coefficients of activation time constant) were obtained using DAPC data from the "hybrid" neuron comprising the in silico model cell and the CHO cell expressing $\mathrm{Na}_{v} 1.2$. 


\section{DISCUSSION}

Biophysically realistic modeling of neuronal systems is limited by the quality of the modeling parameters and the complexity of the model. Typically, ion channel conductance parameters are obtained from analysis of voltage clamp data $(1,6,13,35)$, however $\mathrm{VC}$ analysis is time consuming and complete exploration of conductance parameters across a range of physiologically relevant voltage and temporal domains can be prohibitive. Meanwhile, recent studies indicate that DC has become more accessible with cost effective implementations only requiring basic knowledge in electronics or programming $(36,37)$. Recently developed approaches that use a combination of VC and DC analyses have been used to model the behavior of voltage-gated channels $(11,18)$. Although they provide real-time modeling capability, these studies typically rely on recorded VC data to accurately characterize ion channel conductance parameters. Here we present a novel DAPC-based method that removes this reliance to rapidly and accurately estimate conductance parameters from voltage-gated channels. In fully simulated experiments, we show that for a single cell, approximately $1 s$ of DAPC data is sufficient to estimate conductance parameters and firing properties, in comparison to approximately 15-20 minutes of $\mathrm{VC}$ data required to obtain a similar estimate. Application of this modeling approach to various $\mathrm{Na}_{\mathrm{v}}$ or $\mathrm{K}_{\mathrm{v}}$ conductances generated $\mathrm{HH}$ parameters that could accurately predict experimentally recorded AP firing.

By constraining fitting to only the periods of AP firing, the DAPC modeling approach weights the data collection in favor of physiologically relevant changes in $\mathrm{V}_{\mathrm{m}}$ that can be considered a major factor contributing to the data efficiency compared to VC experiments. One benefit of this method is that we are also optimizing considering the neuronal property, i.e. AP firing, which is most relevant to the goals of computational modeling in this context. In addition, the DAPC method doesn't rely on complex protocols to separate voltage and time dependent gating in ion channels as does VC. Hence the proposed method performed better with ion channels with complex kinetics such as neuronal sodium channels tested here. However, for ion channels with less sophisticated kinetics such as delayed rectifying potassium channels, both VC and DAPC method should perform with similar accuracy.

DAPC-based parametrization is more efficient than VC-based approaches. Specifically, the modelling of ion channel variants and the action of pharmacological agents on neuronal excitability can be significantly improved, which will impact on efforts directed towards precision medicine in neurogenetic disease (38-40). It is difficult to build models using VC methods that reliably predict the impact of drugs on AP firing and DAPC could be readily used to study a range of drugs at various concentrations that would not be feasible with VC methods. Another obvious application of this approach would be the incorporation of this DAPC parameterised HH conductance in complex network models.

To implement this DAPC-based modeling approach, we overcame a series of computational 
challenges. These included the systematic evaluation of methods to most accurately estimate $\mathrm{HH}$ parameters, such as identifying the best data fitting method, designing the ideal stimulation strategy, determining and correcting to minimize the impact of external noise and, finally, to determine the DAPC implementation best suited for this modeling approach.

\subsection{Identifying the best data fitting method}

For DC data fitting, the local gradient-based optimization that has been utilized in the past for fitting DC data (11) was assessed. However, local gradient methods are known to fail to converge to global minima as they can often get trapped in local minima especially when multi-modal fitness functions, required for complex neuronal systems, are used. In fitting DC data using local gradient methods, Milescu et al. (11) relied on a priori VC data to obtain quality fits. Here, we employed global stochastic-based optimization that enabled fitting of DAPC data without the need for prior knowledge from VC data to initialize and constrain parameter search space thus avoiding being caught in local minima on account of ill conditioned parameter values and being well suited to searching large parameter spaces (25). Global optimization methods have been successfully utilized in neuron modeling in recent studies and, in particular, for current clamp data fitting and neural network modeling that inherently demands large parameter space searching $(16,17,41)$. We opted for the SaDE optimization algorithm (25) as it is well suited to continuous landscape problems and it adapts the process for new parameter generation at different stages of the optimization to improve convergence performance.

\subsection{Designing the ideal stimulation strategy}

The synaptic noise model based on the Ornstein-Uhlenbeck process produced the most efficient stimulation strategyfor accurately determining $\mathrm{HH}$ parameters for several reasons. First, stochastic stimuli such as synaptic, pink and white noise provide transient inputs to the neuronal system that are essential to efficiently explore diverse output states, whereas deterministic step current stimuli by definition reduce information flow as they explore a more limited feature space and often repetitively sample similar envelopes of behavior. Second, the spectra of synaptic and pink noise are more closely matched to the characteristic impedance spectrum of biological membranes and the resident ion channels than white noise stimuli (42), where much of the energy of the input signal would be naturally filtered. Ornstein-Uhlenbeck-based synaptic noise was ultimately chosen over pink noise as there is more biological equivalence than with random pink noise in generating functions that can be exploited for various downstream modeling applications. 


\subsection{Assessing the effect of external noise}

The impact of signal noise is a major factor in determining the stability of a DAPC system and can only be controlled to limits set by instrumentation. Even in cases where SNR allows for stability during DAPC recording, there is an impact of noise on the accuracy of firing. Therefore, DAPC data noise also impacts the quality of the fitting for post-acquisition parameter extraction. To limit the impact of noise on the parameter estimation, a two-step process was used. This process produced a marked improvement in parameter accuracy and should be more efficient with noisy experimental data compared to conventional one-step methods. The benefits of two-step method compared to standard optimization need to be further explored across a range of SNRs to better understand benefits and potential weaknesses. Further analysis of the impact of noise on the quality of parameter estimation suggests that for HH parameterestimation, the SNR of the DAPC system should exceed $52 \mathrm{~dB}$ and for general DAPC mode experiments that will not be used for post hoc modeling a SNR greater than $50 \mathrm{~dB}$ is recommended. These are readily obtainable SNRs that can be achieved with good shielding and instrumentation.

\subsection{DAPC implementation}

The three key factors that need to be considered when implementing a DAPC system for modeling approaches are speed,accuracy and flexibility in implementation. These factors are often interrelated. The DAPC systems can be categorized in terms of types of processor and methods of estimating voltage dependent parameters (43). Due to more accuracy in the calculation-based parameter estimation method $(44,45)$ when compared to memory-based systems which employ lookup tables (46), we used the calculation-based approach at the expense of speed in the present study. Moreover, the dedicated processor DAPC implementations $(45,46)$ are typically faster than PC-based implementations $(44,47)$, hence used in this study. The processor could accurately integrate a single compartment model of two ionchannels and synaptic stimuli up to speeds of 140 $\mathrm{kHz}$ and was sufficient for the proposed modeling approach.

\subsection{Considerations for future studies}

A further advantage of DAPC over VC is being able to explore the parameter space of the conductance under investigation using a range of in silico models within the feedback loop. Although the present study did not undertake a systematic evaluation of the impact of the choice of in silico model on the efficiency and accuracy of parametrization, this is an important future endeavor. Tailoring of the in silico feedback model that acknowledges the downstream application of the 
parameters should provide tangible benefits. For example, if the conductance parameters will be used in a large-scale cortical neuronal network model, then using DAPC feedback models of neurons and compartments that are relevant to these brain regions should provide parameters that have been appropriately optimized.

In the present study only fast inactivation properties of sodium channels were modeled and it is very clear that slow inactivation of sodium channels is physiologically and pathologically relevant (4850). The DAPC parameterization method described in this study is readily adaptable and extensible to accommodate a broader range of physiological, pathological and pharmacological conditions. Adaptation of our DAPC modeling approach to account for slow inactivation would entail an investigation of the stationariness of channel behavior under DAPC mode recording as well as incorporation of slow inactivation gates to the standard HH formalism. In addition to exploration of greater temporal envelopes, the exploration of pathological voltage dynamics could be used to assess parameter states during electrical activity seen in epilepsy or other disease conditions. Finally, development of $\mathrm{HH}$ parameters for drug bound states would allow for future in silico modeling of largescale brain network and prediction of drug impacts on emergent behavior that could be relevant to a variety of neurological and psychiatric conditions. With the advances in computation performance it should be possible for parameter estimation to occur in real time. Real-time parameters could be assessed interactively in larger scale models which could assist in evaluation of ion channel mutations and selection of drugs for precision medicine application for a broader range of neurogenetic conditions. 


\section{CONCLUSION}

Modeling extends our understanding of neuronal function. Current methods of modeling neurons utilize data derived from voltage or current clamp experiments. These methods require significant experimental effort and computation that limits their use to specific problems. We proposed an improved approach to parameterize $\mathrm{HH}$ conductance equations using data obtained entirely from brief periods of dynamic action potential clamp recording. The proposed DAPC approach provides a significant experimental time savings over VC based approaches without any penalty on accuracy, freeing resources to broaden the use of modeling to more laboratories and for a wider cross section of problems such as variant analysis in genetic disease and rational development of drugs targeted at precise disease mechanisms. 


\section{AUTHOR CONTRIBUTIONS}

Y.D., S.H. and S.P. designed research; Y.D. and G.B. performed research; G.B. and D.K. contributed new reagents/analytictools; Y.D., S.H. and S.P. analyzed data; and Y.D., G.B., D.K., I.C.F., S.H., and S.P. wrote the paper. 


\section{ACKNOWLEDGMENTS}

Y.D. is fully supported by The University of Melbourne PhD scholarship scheme. This work is also partially funded by Australian Research Council grant DP150103512 to S.H. and NHMRC Research Fellowship grant GNT1005050 and ARC Australian Research Council Centre of Excellence for Integrative Brain Function grant CE140100007 to S.P. S.P. is also a co-founder and equity holder of RogCon, Inc., Miami, USA, and Praxis Precision Medicines, biotechnology companies focused on drug research, discovery and development for ion channel disorders including SCN2A. G.B. was funded by RogCon, Inc. 


\section{COMPETING INTERESTS}

Steven Petrou is co-founder and equity holder of RogCon, Inc., Miami, USA, and Praxis Precision Medicines, biotechnology companies focused on drug research, discovery and development for ion channel disorders including SCN2A. Géza Berecki was funded by RogCon, Inc. 


\section{REFERENCES}

1. Thomas, E. A., R. Xu, and S. Petrou. 2007. Computational analysis of the R85C and R85H epilepsy mutations in Na+ channel beta1 subunits. Neuroscience 147(4):1034-1046.

2. Hu, W., C. Tian, T. Li, M. Yang, H. Hou, and Y. Shu. 2009. Distinct contributions of Na(v)1.6 and $\mathrm{Na}(\mathrm{v}) 1.2$ in action potential initiation and backpropagation. Nat Neurosci 12(8):996-1002.

3. Gonzalez-Ramirez, L. R., O. J. Ahmed, S. S. Cash, C. E. Wayne, and M. A. Kramer. 2015. A biologically constrained, mathematical model of cortical wave propagation preceding seizure termination. PLoS Comput Biol 11(2):e1004065.

4. Markram, H., E. Muller, S. Ramaswamy, M. W. Reimann, M. Abdellah, C. A. Sanchez, A. Ailamaki, L. Alonso-Nanclares, N. Antille, S. Arsever, G. A. Kahou, T. K. Berger, A. Bilgili, N. Buncic, A. Chalimourda, G. Chindemi, J. D. Courcol, F. Delalondre, V. Delattre, S. Druckmann, R. Dumusc, J. Dynes, S. Eilemann, E. Gal, M. E. Gevaert, J. P. Ghobril, A. Gidon, J. W. Graham, A. Gupta, V. Haenel, E. Hay, T. Heinis, J. B. Hernando, M. Hines, L. Kanari, D. Keller, J. Kenyon, G. Khazen, Y. Kim, J. G. King, Z. Kisvarday, P. Kumbhar, S. Lasserre, J. V. Le Be, B. R. Magalhaes, A. Merchan-Perez, J. Meystre, B. R. Morrice, J. Muller, A. Munoz-Cespedes, S. Muralidhar, K. Muthurasa, D. Nachbaur, T. H. Newton, M. Nolte, A. Ovcharenko, J. Palacios, L. Pastor, R. Perin, R. Ranjan, I. Riachi, J. R. Rodriguez, J. L. Riquelme, C. Rossert, K. Sfyrakis, Y. Shi, J. C. Shillcock, G. Silberberg, R. Silva, F. Tauheed, M. Telefont, M. Toledo-Rodriguez, T. Trankler, W. Van Geit, J. V. Diaz, R. Walker, Y. Wang, S. M. Zaninetta, J. DeFelipe, S. L. Hill, I. Segev, and F. Schurmann. 2015. Reconstruction and Simulation of Neocortical Microcircuitry. Cell 163(2):456-492.

5. Wu, X. X., C. Yao, and J. Shuai. 2015. Enhanced multiple vibrational resonances by Na+ and K+ dynamics in a neuron model. Sci Rep 5:7684.

6. Hodgkin, A. L., and A. F. Huxley. 1952. A quantitative description of membrane current and its application to conduction and excitation in nerve. The Journal of physiology 117(4):500-544.

7. Migliore, M., D. A. Hoffman, J. C. Magee, and D. Johnston. 1999. Role of an A-type K+ conductance in the back-propagation of action potentials in the dendrites of hippocampal pyramidal neurons. Journal of computational neuroscience 7(1):5-15.

8. Knox, A. T., T. Glauser, J. Tenney, W. W. Lytton, and K. Holland. 2018. Modeling pathogenesis and treatment response in childhood absence epilepsy. Epilepsia 59(1):135-145.

9. Glazebrook, P. A., A. N. Ramirez, J. H. Schild, C.-C. Shieh, T. Doan, B. A. Wible, and D. L. Kunze. 2002. Potassium channels Kv1.1, Kv1.2 and Kv1.6 influence excitability of rat visceral sensory neurons. The Journal of Physiology 541(2):467-482.

10. Gurkiewicz, M., and A. Korngreen. 2007. A numerical approach to ion channel modelling using wholecell voltage-clamp recordings and a genetic algorithm. PLoS Comput Biol 3(8):e169.

11. Milescu, L. S., T. Yamanishi, K. Ptak, M. Z. Mogri, and J. C. Smith. 2008. Real-time kinetic modeling of voltage-gated ion channels using dynamic clamp. Biophys J 95(1):66-87.

12. Bahl, A., M. B. Stemmler, A. V. Herz, and A. Roth. 2012. Automated optimization of a reduced layer 5 pyramidal cell model based on experimental data. J Neurosci Methods 210(1):22-34.

13. Michel, C. B., C. Azevedo Coste, G. Desmadryl, J. L. Puel, J. Bourien, and B. P. Graham. 2015. Identification and modelling of fast and slow Ih current components in vestibular ganglion neurons. Eur J Neurosci 42(10):2867-2877.

14. Baranauskas, G., T. Tkatch, K. Nagata, J. Z. Yeh, and D. J. Surmeier. 2003. Kv3.4 subunits enhance the repolarizing efficiency of Kv3.1 channels in fast-spiking neurons. Nat Neurosci 6(3):258-266. 
15. Cestele, S., A. Labate, R. Rusconi, P. Tarantino, L. Mumoli, S. Franceschetti, G. Annesi, M. Mantegazza, and A. Gambardella. 2013. Divergent effects of the T1174S SCN1A mutation associated with seizures and hemiplegic migraine. Epilepsia 54(5):927-935.

16. Brookings, T., M. L. Goeritz, and E. Marder. 2014. Automatic parameter estimation of multicompartmental neuron models via minimization of trace error with control adjustment. J Neurophysiol 112(9):2332-2348.

17. Meliza, C. D., M. Kostuk, H. Huang, A. Nogaret, D. Margoliash, and H. D. Abarbanel. 2014. Estimating parameters and predicting membrane voltages with conductance-based neuron models. Biol Cybern 108(4):495-516.

18. Tomaiuolo, M., R. Bertram, G. Leng, and J. Tabak. 2012. Models of electrical activity: calibration and prediction testing on the same cell. Biophys J 103(9):2021-2032.

19. Berecki, G., J. G. Zegers, A. O. Verkerk, Z. A. Bhuiyan, B. de Jonge, M. W. Veldkamp, R. Wilders, and A. C. van Ginneken. 2005. HERG channel (dys)function revealed by dynamic action potential clamp technique. Biophys J 88(1):566-578.

20. Sharp, A. A., M. B. O'Neil, L. F. Abbott, and E. Marder. 1993. Dynamic clamp: computer-generated conductances in real neurons. Journal of neurophysiology 69(3):992-995.

21. Carbonell-Pascual, B., E. Godoy, A. Ferrer, L. Romero, and J. M. Ferrero. 2016. Comparison between Hodgkin-Huxley and Markov formulations of cardiac ion channels. Journal of theoretical biology 399:92-102.

22. Schultheiss, N. W., J. R. Edgerton, and D. Jaeger. 2010. Phase response curve analysis of a full morphological globus pallidus neuron model reveals distinct perisomatic and dendritic modes of synaptic integration. J Neurosci 30(7):2767-2782.

23. Fujita, T., T. Fukai, and K. Kitano. 2012. Influences of membrane properties on phase response curve and synchronization stability in a model globus pallidus neuron. J Comput Neurosci 32(3):539-553.

24. Dougalis, A. G., G. A. C. Matthews, B. Liss, and M. A. Ungless. 2017. Ionic currents influencing spontaneous firing and pacemaker frequency in dopamine neurons of the ventrolateral periaqueductal gray and dorsal raphe nucleus (vlPAG/DRN): A voltage-clamp and computational modelling study. J Comput Neurosci 42(3):275-305.

25. Qin, A. K., V. L. Huang, and P. N. Suganthan. 2009. Differential Evolution Algorithm With Strategy Adaptation for Global Numerical Optimization. IEEE Transactions on Evolutionary Computation 13(2):398-417.

26. Destexhe, A., M. Rudolph, J.-M. Fellous, and T. J. Sejnowski. 2001. Fluctuating synaptic conductances recreate in vivo-like activity in neocortical neurons. Neuroscience 107(1):13-24.

27. zhivomirov, H. Pink, Red, Blue and Violet Noise Generation with Matlab Implementation 24/10/2018). https://au.mathworks.com/matlabcentral/fileexchange/42919-pink-red-blue-and-violetnoise-generation-with-matlab

28. Berecki, G., K. B. Howell, Y. H. Deerasooriya, M. R. Cilio, M. K. Oliva, D. Kaplan, I. E. Scheffer, S. F. Berkovic, and S. Petrou. 2018. Dynamic action potential clamp predicts functional separation in mild familial and severe de novo forms of SCN2A epilepsy. Proc Natl Acad Sci U S A 115(24):E5516-E5525.

29. Carnevale, N. T., and M. L. Hines. 2006. The NEURON book. Cambridge University Press.

30. Wang, W., F. Xiao, X. Zeng, J. Yao, M. Yuchi, and J. Ding. 2012. Optimal estimation of ion-channel kinetics from macroscopic currents. PLoS One 7(4):e35208. 
31. Lankarany, M., W.-P. Zhu, M. Swamy, and T. Toyoizumi. 2013. Blind deconvolution of HodgkinHuxley neuronal model. In Engineering in Medicine and Biology Society (EMBC), 2013 35th annual international conference of the IEEE. IEEE. 3941-3944.

32. Gurkiewicz, M., A. Korngreen, S. G. Waxman, and A. Lampert. 2011. Kinetic modeling of Nav1.7 provides insight into erythromelalgia-associated F1449V mutation. J Neurophysiol 105(4):1546-1557.

33. Hay, E., F. Schurmann, H. Markram, and I. Segev. 2013. Preserving axosomatic spiking features despite diverse dendritic morphology. J Neurophysiol 109(12):2972-2981.

34. Groenendaal, W., F. A. Ortega, A. R. Kherlopian, A. C. Zygmunt, T. Krogh-Madsen, and D. J. Christini. 2015. Cell-specific cardiac electrophysiology models. PLoS Comput Biol 11(4):e1004242.

35. Csercsik, D., K. M. Hangos, and G. Szederkényi. 2012. Identifiability analysis and parameter estimation of a single Hodgkin-Huxley type voltage dependent ion channel under voltage step measurement conditions. Neurocomputing 77(1):178-188.

36. Yang, Y., T. Adowski, B. Ramamurthy, A. Neef, and M. A. Xu-Friedman. 2015. High-speed dynamicclamp interface. J Neurophysiol 113(7):2713-2720.

37. Desai, N. S., R. Gray, and D. Johnston. 2017. A Dynamic Clamp on Every Rig. eNeuro 4(5).

38. Kuo, C.-C., and B. P. Bean. 1994. Slow binding of phenytoin to inactivated sodium channels in rat hippocampal neurons. Molecular Pharmacology 46(4):716-725.

39. Carvill, G. L., S. B. Heavin, S. C. Yendle, J. M. McMahon, B. J. O'Roak, J. Cook, A. Khan, M. O. Dorschner, M. Weaver, S. Calvert, S. Malone, G. Wallace, T. Stanley, A. M. Bye, A. Bleasel, K. B. Howell, S. Kivity, M. T. Mackay, V. Rodriguez-Casero, R. Webster, A. Korczyn, Z. Afawi, N. Zelnick, T. Lerman-Sagie, D. Lev, R. S. Moller, D. Gill, D. M. Andrade, J. L. Freeman, L. G. Sadleir, J. Shendure, S. F. Berkovic, I. E. Scheffer, and H. C. Mefford. 2013. Targeted resequencing in epileptic encephalopathies identifies de novo mutations in CHD2 and SYNGAP1. Nat Genet 45(7):825-830.

40. Epi, K. C., P. Epilepsy Phenome/Genome, A. S. Allen, S. F. Berkovic, P. Cossette, N. Delanty, D. Dlugos, E. E. Eichler, M. P. Epstein, T. Glauser, D. B. Goldstein, Y. Han, E. L. Heinzen, Y. Hitomi, K. B. Howell, M. R. Johnson, R. Kuzniecky, D. H. Lowenstein, Y. F. Lu, M. R. Madou, A. G. Marson, H. C. Mefford, S. Esmaeeli Nieh, T. J. O'Brien, R. Ottman, S. Petrovski, A. Poduri, E. K. Ruzzo, I. E. Scheffer, E. H. Sherr, C. J. Yuskaitis, B. Abou-Khalil, B. K. Alldredge, J. F. Bautista, S. F. Berkovic, A. Boro, G. D. Cascino, D. Consalvo, P. Crumrine, O. Devinsky, D. Dlugos, M. P. Epstein, M. Fiol, N. B. Fountain, J. French, D. Friedman, E. B. Geller, T. Glauser, S. Glynn, S. R. Haut, J. Hayward, S. L. Helmers, S. Joshi, A. Kanner, H. E. Kirsch, R. C. Knowlton, E. H. Kossoff, R. Kuperman, R. Kuzniecky, D. H. Lowenstein, S. M. McGuire, P. V. Motika, E. J. Novotny, R. Ottman, J. M. Paolicchi, J. M. Parent, K. Park, A. Poduri, I. E. Scheffer, R. A. Shellhaas, E. H. Sherr, J. J. Shih, R. Singh, J. Sirven, M. C. Smith, J. Sullivan, L. Lin Thio, A. Venkat, E. P. Vining, G. K. Von Allmen, J. L. Weisenberg, P. Widdess-Walsh, and M. R. Winawer. 2013. De novo mutations in epileptic encephalopathies. Nature 501(7466):217-221.

41. Carlson, K. D., J. M. Nageswaran, N. Dutt, and J. L. Krichmar. 2014. An efficient automated parameter tuning framework for spiking neural networks. Front Neurosci 8:10.

42. Carandini, M., F. Mechler, C. S. Leonard, and J. A. Movshon. 1996. Spike train encoding by regularspiking cells of the visual cortex. Journal of Neurophysiology 76(5):3425-3441.

43. Prinz, A. A., L. F. Abbott, and E. Marder. 2004. The dynamic clamp comes of age. Trends Neurosci 27(4):218-224.

44. Dorval, A. D., D. J. Christini, and J. A. White. 2001. Real-time linux dynamic clamp: a fast and flexible way to construct virtual ion channels in living cells. Annals of biomedical engineering 29(10):897-907.

45. Mak, T. S., G. Rachmuth, K. P. Lam, and C. S. Poon. 2006. A component-based FPGA design framework for neuronal ion channel dynamics simulations. IEEE Trans Neural Syst Rehabil Eng 14(4):410-418. 
46. Bast, W., D. Dellavale, and F. Bonetto. 2011. FPGA implementation of a "dynamic-clamp" system. In Programmable Logic (SPL), 2011 VII Southern Conference on. IEEE. 167-172.

47. Nowotny, T., A. Szucs, R. D. Pinto, and A. I. Selverston. 2006. StdpC: a modern dynamic clamp. J Neurosci Methods 158(2):287-299.

48. Rudy, B. 1978. Slow inactivation of the sodium conductance in squid giant axons. Pronase resistance. The Journal of physiology 283(1):1-21.

49. Vilin, Y. Y., and P. C. Ruben. 2001. Slow inactivation in voltage-gated sodium channels. Cell biochemistry and biophysics 35(2):171-190.

50. Wang, Y., S. M. Wilson, J. M. Brittain, M. S. Ripsch, C. Salomé, K. D. Park, F. A. White, R. Khanna, and H. Kohn. 2011. Merging structural motifs of functionalized amino acids and $\alpha$-aminoamides results in novel anticonvulsant compounds with significant effects on slow and fast inactivation of voltage-gated sodium channels and in the treatment of neuropathic pain. ACS chemical neuroscience 2(6):317-332. 